\title{
Dipolar magnetic order in crystals of molecular nanomagnets
}

\author{
Fernando Luis \\ Instituto de Ciencia de Materiales de Aragón, C.S.I.C. - Universidad de Zaragoza, \\ and Dpto. de Física de la Materia Condensada, Universidad de Zaragoza, E-50009 \\ Zaragoza, Spain, fluis@unizar.es
}

\begin{abstract}
This chapter reviews experimental studies of long-range dipolar magnetic order, of which few examples exist in Nature, in crystals of single-molecule magnets. Quantum annealing by a transverse magnetic field enables one to explore the ground state of highly anisotropic SMMs, such as $\mathrm{Mn}_{12}$ and $\mathrm{Fe}_{8}$, both of which order ferromagnetically below $T_{\mathrm{c}}=0.9 \mathrm{~K}$ and $0.6 \mathrm{~K}$, respectively. $\mathrm{In}_{\mathrm{Mn}_{12}}$ acetate, molecular tilts caused by the disorder in the orientations of some solvent molecules affect dramatically the character of the field-induced transition, which agrees with the predictions of the random-field Ising model. The existence of a quantum critical point has been shown in crystals of $\mathrm{Fe}_{8}$ clusters, which are among the best realizations of the archetypical quantum Ising model in a transverse magnetic field.
\end{abstract}

\subsection{Introduction}

Dipolar interactions are ubiquitous in Nature. A dipolar magnetic moment $\boldsymbol{\mu}_{\mathbf{i}}$, e.g. a magnetic ion, generates a magnetostatic field that affects other dipoles $\boldsymbol{\mu}_{\mathbf{j}}$ located in its surroundings. The coupling energy between any pair of dipoles separated by a position vector $\boldsymbol{r}_{\mathbf{i j}}$ can be expressed as follows

$$
\mathcal{H}_{\mathrm{dip}, \mathrm{ij}}=-\left[\frac{3\left(\boldsymbol{\mu}_{\mathrm{i}} r_{\mathrm{ij}}\right)\left(\boldsymbol{\mu}_{\mathrm{j}} \boldsymbol{r}_{\mathrm{ij}}\right)}{r_{\mathrm{ij}}{ }^{5}}-\frac{\mu_{\mathrm{i}} \mu_{\mathrm{j}}}{r_{\mathrm{ij}}{ }^{3}}\right]
$$

Dipolar interactions are known to affect the physical behavior of magnetic materials in a number of ways. They often dominate the line broadening of resonance spectra measured on paramagnets [1]. In magnetically ordered materials, an important manifestation is the formation of magnetic domains pointing along different orientations [2]. However, dipolar interactions often play but a minor role in determining the intrinsic magnetic structure. In order to better understand why this is the case, let us consider a specific and simple example: pure metallic iron. Iron is a ferromagnet below a Curie temperature $T_{\mathrm{c}}=1046 \mathrm{~K}$. The typical dipolar energy between nearest neighbor Fe atoms 


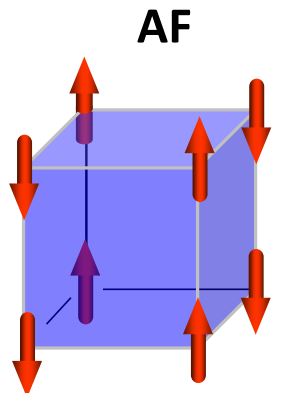

Simple cubic



Body centered cubic Face centered cubic

Fig. 1.1. Luttinger and Tisza [4] solutions for the ground-state configurations of interacting magnetic dipoles located on each of the three Bravais cubic lattices

amounts to approximately $0.5 \mathrm{~K}$. Clearly, dipolar interactions are much weaker than exchange interactions, of quantum mechanical origin, and the later drive the onset of magnetic order in iron as well and in the vast majority of magnetic materials. The same argument explains why it is so difficult to find systems in Nature showing pure dipolar magnetic order. Even relatively weak exchange couplings, difficult to avoid, might dominate over dipolar interactions.

\section{Brief survey of theoretical studies}

Not surprisingly, the first steps in the study of dipolar magnetism were almost exclusively of a theoretical nature. Compared with the situation met when exchange interactions are dominant, the problem statement is appealingly simple. Interactions are known, and given by Eq. (1.1), and all that needs to be done is to minimize the free energy of a given lattice of dipoles over all possible configurations. However, its numerical solution is complicated by the long-range character of dipolar interactions. Early attempts consisted of numerical calculations (carried out without the aid of a computer!) of the energies of some configurations of classical dipoles located in simple lattices [3]. It was not until 1946 that J. M. Luttinger and L. Tisza found a rigorous method that enables finding the ground state configurations for simple, bodycentered, and face-centered cubic lattices [4]. This method was later extended to cover lattices with up to two equivalent dipoles per unit cell [5] and to even more complex lattices in the case of strongly anisotropic (Ising-like) dipoles [6]. The ordering of dipolar Ising crystals was reanalyzed in [7], where a simple expression for the interaction energy between chains of spins pointing along their anisotropy axes was derived. Finally, the ordering temperatures of some materials have been determined by Monte Carlo calculations [7-9]. 


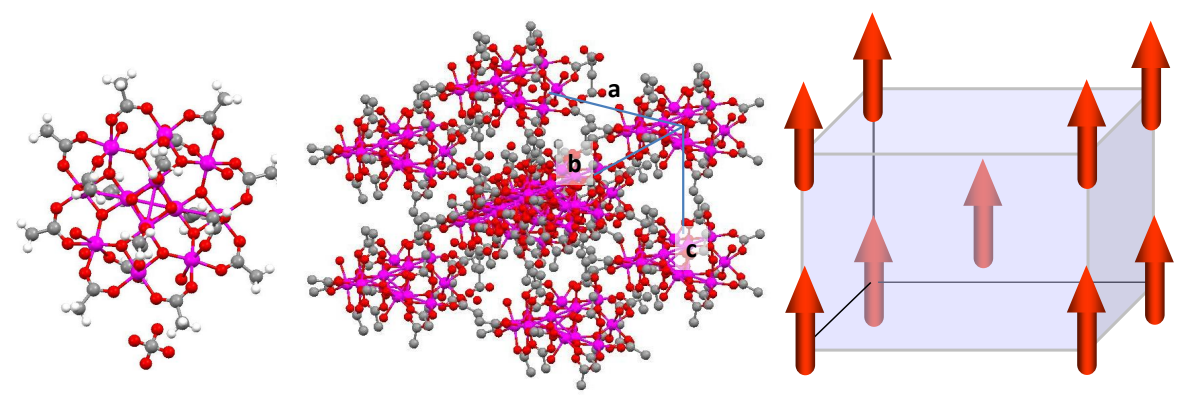

Fig. 1.2. Molecular structure of $\mathrm{Mn}_{12}$ acetate (left) and molecular packing in the tetragonal unit cell (center). This molecular crystal provides a close approximation to a tetragonal lattice of strongly anisotropic (Ising-like) spins, coupled via dipoledipole interactions (right)

It follows from these results that the ground state configurations are dictated by lattice symmetry. In cubic systems (see Fig. 1.1), for instance, lattices with low coordination numbers (diamond and simple cubic lattices) order antiferromagnetically, whereas face centered and body centered lattices are ferromagnetic. The same argument applies, within a certain range of parameters (determined by the ratio $c / a$ ), for tetragonal and hexagonal lattices $[7,10]$. The existence of dipolar ferromagnetism was, however, questioned by Luttinger and Tisza themselves, and thought to depend upon the shape of the specimen [4]. The argument is that the onset of a spontaneous magnetization gives rise, in any finite sample, to an additional increase in magnetostatic energy that depends on its demagnetizing tensor. The existence of a welldefined ground state for macroscopic lattices at zero magnetic field, independent of the specimen's shape, was demonstrated more than 20 years latter by R. B. Griffiths [11]. This result suggests that dipolar ferromagnets, like any other ferromagnetic material, tend to subdivide into magnetic domains below $T_{\mathrm{c}}$ [12]. Some other important theoretical results worth mentioning here are the existence of important zero-point fluctuations [13] and the prediction that three-dimensional dipolar lattices provide close approximations of mean-field models. In particular, the marginal dimensionality for mean-field behavior is $d^{*}=3$ in an Ising dipolar ferromagnet [14].

\section{Experimental realizations: single molecule magnets}

For the reasons mentioned above, experimental realizations of dipolar lattices are scarce, even today. Best-known examples are provided by crystals of lanthanide-based compounds [15-22]. Exchange interactions between lanthanide ions are weak, on account of the localization of $4 f$ electrons. In lattices 
with sufficiently separated ions, dipolar interactions might therefore become of comparable and even dominating strength. In these cases, ordering temperatures are often very low, typically below $100 \mathrm{mK}$. An outstanding exception is represented by $\mathrm{LiHoF}_{4}$, which orders ferromagnetically at $T_{\mathrm{c}}=1.54 \mathrm{~K}[15,18]$. However, in the later case the underlying physics is somewhat complicated by the existence of non-negligible exchange interactions and rather strong hyperfine couplings $[23,24]$.

Crystals of molecular nanomagnets [25-29] are suitable candidate materials to investigate magnetic order of pure dipolar lattices (see Fig. 1.2). Each of these single-molecule magnets (SMMs) is an electrically neutral entity, in which the magnetic core is surrounded, thus also isolated from its neighbors, by a shell of organic ligands. Many of these molecules have large spins (e.g. $S=10$ for $\mathrm{Mn}_{12}$ and $\mathrm{Fe}_{8}$ clusters), and therefore large magnetic moments $\mu=g \mu_{\mathrm{B}} S$, where $g$ is the molecular gyromagnetic factor. Dipolar interactions are then relatively strong and often dominate over the very weak, if present at all, exchange interactions. Ordering temperatures are expected to be of the order of $0.5 \mathrm{~K}$ or even higher [7], which considerably simplifies the experimental study of the magnetic phase transitions by a variety of techniques, including heat capacity, magnetic susceptibility, and magnetic neutron diffraction.

From a fundamental point of view, these crystals provide close to ideal realizations of physical models, such as the quantum Ising model [30], of broad interest for physics. Dipolar interactions also affect the spin dynamics. This effect is particularly important at very low temperatures, when spin flips occur predominantly via pure tunneling processes. Under these conditions, dipolar bias fields energetically detune states between which tunneling takes place, and magnetic relaxation becomes a collective phenomenon [31]. It follows then that not only the equilibrium state, but also the rate at which this state is attained strongly depend on the onset of magnetic order below $T_{\mathrm{c}}$ [32]. Knowing the equilibrium magnetic state is therefore a necessary pre-requisite to fully understand magnetic relaxation and quantum tunneling phenomena observed at very low temperatures [33-39]. A particularly attractive question is the competition between dipolar interactions, typically weak, and quantum fluctuations, which are strong in molecular nanomagnets and can be made even stronger via the application of an external magnetic field, eventually leading to a quantum phase transition $[40,41]$.

The information gained via these studies can also be of relevance to other scientific fields and even to applications. In many aspects (e.g., the existence of magnetic memory effects at sufficiently low temperatures, associated with a strong magnetic anisotropy), crystals of SMMs are equivalent to ordered and monodisperse arrays of magnetic nanoparticles. The study of dipolar interactions in the former provides useful information on the nature of the collective magnetic response of coupled nanomagnets [42]. The onset of long-range magnetic order reduces the entropy of the spin lattice, which rapidly vanishes below $T_{\mathrm{c}}$. This effect ultimately limits the lowest temperature attainable by adiabatic demagnetization methods. Molecular nanomagnets are among the 
best magnetic coolers at liquid Helium temperatures [43]. The study of dipolar ordering in these materials, and how it depends on crystal symmetry and magnetic anisotropy, is then of practical interest for magnetic refrigeration technologies.

\section{Outline of the chapter}

The present chapter is written from an experimental perspective. Its aim is mainly to show, with the help of examples, the existence of dipolar order in some of the most famous single-molecule magnets, in particular $\mathrm{Mn}_{12}$ acetate and $\mathrm{Fe}_{8}$, how these phenomena have been experimentally uncovered, and what physics can be learned from it. Section 1.2 provides a very basic theoretical background on the interactions that play a role in determining the physical behavior of SMMs lattices, and their respective effects. This section introduces also mean-field approximations, which are simple and therefore especially convenient to analyze the results of experiments. Section 1.3 discusses one of the most serious difficulties faced by such experiments and which is related to the slow relaxation of molecular nanomagnets. This section also shows how measurements of the magnetization dynamics and hysteresis can be used to estimate the effective intermolecular interaction fields. Sections 1.4 to 1.6 describe the results of experimental studies of magnetic order performed on several molecular materials. The experiments performed on $\mathrm{Mn}_{12}$ (section 1.5) and $\mathrm{Fe}_{8}$ (section 1.6) illustrate that highly interesting physical phenomena result from the competition between dipolar interactions and transverse magnetic fields. The last section 1.7 summarizes the main conclusions and suggests possible evolutions of this research field.

Most of the results described in this chapter refer to work done, and published [44-49], in the course of the past decade. Yet, it contains a few original

aspects too, in particular the determination of the interaction fields in $\mathrm{Mn}_{12}$ acetate that is included in section 1.3. Also, the interpretation of some of the experimental results are re-examined on the basis of subsequent theoretical [10] and experimental [50] developments.

\subsection{Theoretical background}

\subsubsection{Spin Hamiltonian}

The spin Hamiltonian of a lattice of SMMs coupled via dipolar interactions can be written as follows

$$
\mathcal{H}=\frac{1}{2} \sum_{i} \sum_{j \neq i} \mathcal{H}_{\mathrm{dip}, \mathrm{ij}}+\sum_{i} \mathcal{H}_{0, i}+\sum_{i} \mathcal{H}_{Z, i}
$$

where the dipolar interaction Hamiltonian $\mathcal{H}_{\text {dip }, \mathrm{ij}}$ is given by Eq.(1.1), $\mathcal{H}_{0, \mathrm{i}}$ gives the magnetic anisotropy of each isolated molecule 

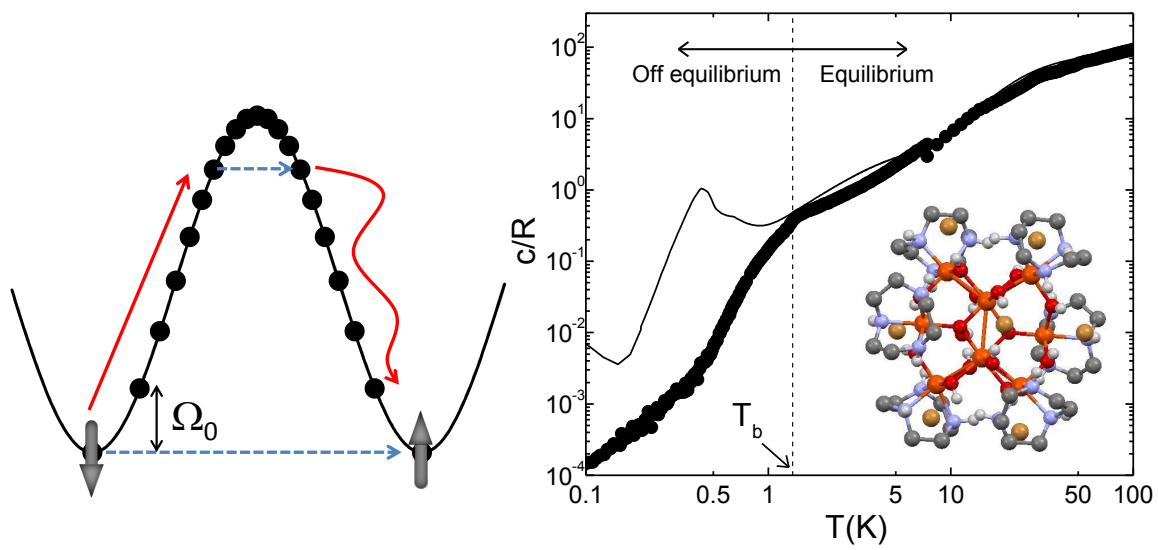

Fig. 1.3. Left: Structure of magnetic energy levels of a generic SMM. The spin reversal can take place via a thermally activated mechanism or via pure quantum tunneling processes. Right: Zero-field specific heat of $\mathrm{Fe}_{8}$, whose molecular structure is shown in the inset. Above the blocking temperature $T_{\mathrm{b}}$, the experimental data (dots) agree with the equilibrium specific heat, obtained from Monte Carlo calculations (solid line). Below $T_{\mathrm{b}}$, it decreases rapidly, showing no hint of the phase transition to long-range magnetic order

$$
\mathcal{H}_{0}=-D S_{z}^{2}+B S_{z}^{4}+E\left(S_{x}^{2}-S_{y}^{2}\right)+\frac{C}{2}\left(S_{+}^{4}+S_{-}^{4}\right)+\ldots
$$

where $D, B, E, C, \ldots$ are anisotropy parameters, and

$$
\mathcal{H}_{\mathrm{Z}}=-g \mu_{\mathrm{B}} H_{z} S_{z}-g \mu_{\mathrm{B}} H_{\perp}\left(S_{x} \cos \phi+S_{y} \sin \phi\right)
$$

describes the Zeeman interaction with an external magnetic field $\boldsymbol{H}$, having components $H_{z}$ along the anisotropy axis $z$ and $H_{\perp}$ perpendicular to it, where $\phi$ is the azimuthal angle of $\boldsymbol{H}$ in the $x y$ plane.

The zero-field energy level scheme of a generic SMM with Ising-like uniaxial anisotropy (i.e. with $D>0$ and weak higher-order anisotropies) is schematically shown in Fig. 1.3. "Diagonal" terms (i.e. those commuting with $S_{z}$ ) give rise to a classical energy barrier $U_{\mathrm{cl}}=D S^{2}-B S^{4}$, separating spin-up (i.e. eigenstates of $S_{z}$ with eigenvalue $m>0$ ) from spin-down states (with $m<0$ ). Off-diagonal terms (i.e. non-commuting with $S_{z}$ ), induce quantum tunneling between magnetic states $\pm m$, at zero field, and between $m$ and $-m-n$, with $n$ integer, at the "crossing fields"

$$
H_{z, \mathrm{n}}(m)=n\left(D / g \mu_{\mathrm{B}}\right)\left\{1+B\left[m^{2}+(m+n)^{2}\right] / D\right\}
$$

At these fields, the classical degeneracy between the crossing levels is lifted by a finite quantum tunnel splitting $\Delta_{m}$. 
The first term in Eq. (1.2) induces, below a critical temperature $T_{\mathrm{c}}$, a phase transition to a long-range magnetically ordered state, which is mainly determined by crystal symmetry and lattice parameters [7]. Magnetic interactions compete with the polarization induced by an external magnetic field $\boldsymbol{H}$. In addition, and similarly to what happens with exchange-coupled spin systems [51], both the nature of the ensuing magnetic order and $T_{\mathrm{c}}$ are affected by the magnetic anisotropy. A particular case, which is highly relevant to most SMMs, arises when the uniaxial anisotropy is much stronger than dipolar interactions. More specifically, when the zero-field splitting $\Omega_{0} \simeq(2 S-1) D$ that separates the ground and first excited level doublets of Eq. (1.3) is much larger than the characteristic interaction energy, given by $k_{\mathrm{B}} T_{\mathrm{c}}$, the dipolar Hamiltonian (1.1) can be simplified to the following Ising interaction Hamiltonian

$$
\mathcal{H}_{\mathrm{dip}, \mathrm{ij}} \simeq-\left[\frac{3\left(\mu_{\mathrm{i}, \mathrm{z}} z_{\mathrm{ij}}\right)\left(\mu_{\mathrm{j}, \mathrm{z}} z_{\mathrm{ij}}\right)}{r_{\mathrm{ij}}{ }^{5}}-\frac{\mu_{\mathrm{i}, \mathrm{z}} \mu_{\mathrm{j}, \mathrm{z}}}{r_{\mathrm{ij}}{ }^{3}}\right]
$$

involving only $S_{z}$.

Zero-field magnetic ground states and ordering temperatures $T_{\mathrm{c}}$ of some specific lattice symmetries, relevant to some particular SMMs systems, have been determined using Monte Carlo calculations based on the Ising Hamiltonian $(1.6)[7,45,47]$. Results of some of these calculations are listed in Table 1.1 .

\subsubsection{Mean-field approximations}

An even simpler method to treat the effect of interactions is to make use of a mean-field approximation, which is especially well suited to deal with dipolar magnets [14]. For simplicity, in the following I consider a lattice of spins ordering ferromagnetically. Within the mean-field approximation, the spin Hamiltonian (1.2) reduces itself to an effective Hamiltonian for a single spin (say spin $i$ )

Table 1.1. Magnetic ordering temperatures of some crystals of SMMs. The fifth and sixth columns show data calculated, for pure dipolar interactions, using Monte Carlo and mean-field methods, respectively. The seventh column provides experimental data. Na stands for data that are "not available"

\begin{tabular}{lllllll}
\hline System & lattice & spin & $D / k_{\mathrm{B}}(\mathrm{K})$ & $T_{\mathrm{c}}^{\mathrm{MC}}(\mathrm{K})$ & $T_{\mathrm{c}}^{\mathrm{MF}}$ & $T_{\mathrm{c}}^{\exp }(\mathrm{K})$ \\
\hline $\mathrm{Mn}_{4} \mathrm{Me}$ & monoclinic & $9 / 2$ & 0.69 & 0.1 & na & $0.21(2)[45]$ \\
$\mathrm{Mn}_{6}$ & monoclinic & 12 & 0.013 & 0.22 & na & $0.15(1)[44]$ \\
$\mathrm{Fe}_{8}$ & triclinic & 10 & 0.294 & $0.54[7]$ & na & $0.60(5)[49]$ \\
$\mathrm{Mn}_{12}$ ac & tetragonal & 10 & 0.6 & $0.5[7]$ & $0.8[76]$ & $0.9(1) \mathrm{K}[46]$ \\
$\mathrm{Fe}_{17}$ & trigonal & $35 / 2$ & 0.02 & na & na & $0.8[65]$ \\
\hline
\end{tabular}




$$
\mathcal{H}=\mathcal{H}_{0}-g \mu_{\mathrm{B}} H_{z} S_{z}-g \mu_{\mathrm{B}} H_{\perp}\left(S_{x} \cos \phi+S_{y} \sin \phi\right)-J_{\mathrm{eff}}\left\langle S_{z}\right\rangle_{T} S_{z}
$$

where $\left\langle S_{z}\right\rangle_{T}$ is the thermal equilibrium average of $S_{z}$ and

$$
J_{\mathrm{eff}}=\frac{-\left(g \mu_{\mathrm{B}}\right)^{2}}{2} \sum_{j \neq i}\left(\frac{3 z_{\mathrm{ij}}^{2}}{r_{\mathrm{ij}}}-\frac{1}{r_{\mathrm{ij}}{ }^{3}}\right)
$$

is an effective interaction constant. The last term in Eq.(1.7) can also be written as a Zeeman interaction $-g \mu_{\mathrm{B}} H_{\mathrm{eff}, \mathrm{z}} S_{z}$ with a mean-field magnetic bias

$$
H_{\mathrm{eff}, \mathrm{z}}=\frac{J_{\mathrm{eff}}}{g \mu_{\mathrm{B}}}\left\langle S_{z}\right\rangle_{T}
$$

The mean-field Hamiltonian (1.7) is appealing for experimentalists, because it allows a relatively easy comparison to different measurable quantities. Above $T_{\mathrm{c}}$, the intrinsic (i.e. free from demagnetization effects) equilibrium longitudinal magnetic susceptibility $\chi_{\mathrm{i}, \mathrm{zz}}$ follows Curie-Weiss law

$$
\chi_{\mathrm{i}, \mathrm{zz}}=\frac{C}{T-\theta}
$$

where $C$ is the Curie constant and $\theta=T_{\mathrm{c}}$ is the Weiss temperature. Notice that Eq.(1.10) also applies to dipolar lattices ordering antiferromagnetically. In the latter case, however, $\theta<0$. Analytical expressions for $\theta$ and $C$ can be found for specific limiting situations. For instance, when the magnetic anisotropy is very strong as compared to both dipolar interactions and $k_{\mathrm{B}} T$, each molecular spin behaves effectively as a spin- $1 / 2$ system. Under these conditions (i.e. for $D \rightarrow \infty$ ), Eq. (1.7) reduces to an effective spin-1/2 Hamiltonian

$$
\mathcal{H} \simeq-\mu_{\mathrm{B}} S H_{\perp}\left(g_{x} \sigma_{x} \cos \phi+g_{y} \sigma_{y} \sin \phi\right)-g_{z} \mu_{\mathrm{B}} S H_{z} \sigma_{z}-J_{\mathrm{eff}} S^{2}\left\langle\sigma_{z}\right\rangle_{T} \sigma_{z}
$$

where the $\sigma$ 's are Pauli spin operators, $g_{z} \simeq g$, and $g_{x}$ and $g_{y}$ depend on the ratio between off diagonal and diagonal anisotropy parameters (i.e. on $E / D$, $C / D$, etc). The Curie constant and Weiss temperature then read as follows

$$
\begin{aligned}
C & =n \frac{\left(g \mu_{\mathrm{B}} S\right)^{2}}{3 k_{B}} \\
\theta & =\frac{J_{\text {eff }} S^{2}}{k_{\mathrm{B}}}
\end{aligned}
$$

where $n$ is the concentration of molecular spins per unit of volume. The susceptibility $\chi_{\text {powder }}$ of randomly oriented crystals $\chi_{\text {powder }}=(1 / 3)\left(\chi_{\mathrm{i}, \mathrm{xx}}+\chi_{\mathrm{i}, \mathrm{yy}}+\right.$ 
$\left.\chi_{\mathrm{i}, \mathrm{zz}}\right)$. Often, especially close to $T_{\mathrm{c}}, \chi_{\mathrm{i}, \mathrm{zz}} \gg \chi_{\mathrm{i}, \mathrm{xx}}, \chi_{\mathrm{i}, \mathrm{yy}}$. Therefore, the susceptibility of powdered samples also follows Curie-Weiss law (1.10).

Strictly speaking, equation (1.10) applies to the case of an infinitely long cylindrical sample, whose long axis coincides with $z$. For real samples of finite size, demagnetizing effects play a role [52-54]. The susceptibility that is actually measured in a experiment in then approximately given by the following expression

$$
\chi_{z z} \simeq \frac{\chi_{\mathrm{i}, \mathrm{zz}}}{1+\chi_{\mathrm{i}, \mathrm{zz}} \widetilde{N}_{z z}}
$$

where it has been considered, for simplicity, that the $z$ axis corresponds to a principal axis of the demagnetization tensor $\widetilde{N}$. Notice that, at $T=\theta, \chi_{z z \text {,exp }}$ no longer diverges but approaches $\chi_{\max }=1 / N_{z z}$.

An additional attractive feature of mean-field models is that they can readily include effects of quantum fluctuations, induced by either the magnetic anisotropy or transverse magnetic fields [46], and of molecular disorder $[10,50]$, both of which are cumbersome to deal with using Monte Carlo calculations. These effects give rise to interesting physical phenomena and are also particularly relevant to experimental situations met with some molecular crystals, such as those described below in sections 1.5 and 1.6.

\subsection{Dipolar order vs single-molecule magnet behavior}

\subsubsection{Magnetic order and relaxation towards thermal equilibrium}

The above considerations about magnetic ordering apply only provided that spins reach thermal equilibrium, i.e. the state of minimum free energy, below $T_{\mathrm{c}}$. Relaxation to equilibrium is brought about by the coupling of spins with vibrations of the crystal lattice, which acts as a thermal bath [55-57]. The rate depends on the strength of spin-phonon couplings but also on the structure of magnetic energy levels and the nature of the energy eigenstates. Here, the magnetic anisotropy plays a second, very important role. In many of the best-known single-molecule magnets (SMMs), and as it has been described in detail in previous chapters of this book, relaxation becomes in fact hindered at low temperatures by the presence of high anisotropy energy barriers. This question represents, in fact, one of the most serious difficulties encountered in the search for dipolar magnetic order in crystals of SMMs.

For temperatures not much lower than the zero-field splitting $\Omega_{0}$, relaxation to thermal equilibrium proceeds via thermally activated processes, whose characteristic relaxation time $\tau \simeq \tau_{0} \exp \left(U / k_{\mathrm{B}} T\right)$ increases exponentially with decreasing temperature [58]. For any given experimental time $\tau_{\mathrm{e}}$, spins "freeze", i.e. they deviate from thermal equilibrium below a superparamagnetic "blocking" temperature $T_{\mathrm{b}}=U / k_{\mathrm{B}} \ln \left(\tau_{\mathrm{e}} / \tau_{0}\right)$. Here, the pre-factor $\tau_{0}$ gives the order of magnitude of excited levels lifetimes and $U$ is usually smaller 
than $U_{\mathrm{cl}}$ because spins can flip by tunneling via intermediate states [59-62]. Pure ground state tunneling events might provide also an alternative path for the spin system to approach long-range ordering [32]. However, these processes are usually very slow. For instance, tunneling times measured on $\mathrm{Fe}_{8}$ are of order $10^{4} \mathrm{~s}$ [36], while in $\mathrm{Mn}_{12}$ acetate they are probably longer than 2 months [63]. Therefore, often $T_{\mathrm{b}}>T_{\mathrm{c}}$ and the underlying magnetic order remains hidden.

The situation can be best illustrated with the help of a specific example. Figure 1.3 shows the specific heat $c$ of $\mathrm{Fe}_{8}$ measured at zero field $[39,64]$. Monte Carlo simulations predict a maximum in $c$ signalling the onset of ferromagnetic order at $T_{\mathrm{c}} \simeq 0.5 \mathrm{~K}[7]$. However, experimental data deviate from equilibrium already at $T_{\mathrm{b}}=1.3 \mathrm{~K}$, decreasing exponentially with $T$ and showing no evidence whatsoever for the existence of a phase transition.

The search for dipolar order must therefore be oriented towards crystals of molecular nanomagnets with sufficiently fast spin-lattice relaxation, i.e. those having $T_{\mathrm{b}}<T_{\mathrm{c}}$. Funnily, the goal is just the opposite to that of finding singlemolecules with long-lasting magnetic memory, which has been the main stream of activity in this research field. A remarkable intermediate situation was found in crystals of $\mathrm{Fe}_{17} \mathrm{SMMs}$, with a very high spin $S=35 / 2[65,66]$. These clusters can be packed in two different crystal structures, of cubic and trigonal symmetries, respectively. The critical temperatures associated with dipolar magnetic order in these lattices are different, with $T_{\mathrm{c}}$ (cubic) $<\mathrm{T}_{\mathrm{c}}$ (trigonal). In the cubic case, a situation similar to that described above for $\mathrm{Fe}_{8}$ arises, thus the system behaves as a SMM with a blocking temperature $T_{\mathrm{b}} \simeq 0.5 \mathrm{~K}$. In the trigonal case, $T_{\mathrm{c}} \simeq 0.8 \mathrm{~K}$, thus larger than $T_{\mathrm{b}}$. As a result, both the equilibrium heat capacity and magnetic susceptibility show clear indications of the onset of long-range dipolar order. Further examples in which equilibrium conditions can be attained down to sufficiently low temperatures are described in detail in sections 1.4 to 1.6.

\subsubsection{Influence of dipolar interactions on magnetic relaxation and spin tunneling}

Dipolar interactions modify also the nature and rates of magnetic relaxation processes. In the paramagnetic state, magnetic fields $\boldsymbol{H}_{d}$ vary from one lattice point to another. Near a crossing magnetic field, $H_{z, \mathrm{n}}(m)$, the longitudinal $H_{\mathrm{d}, \mathrm{z}}$ acting on a given molecule detunes energetically states $m$ and $-m-$ $n$, which would otherwise be in resonance. The effect is to block quantum tunneling processes between these states, the more so the smaller the ratio $\Delta_{m} / \xi_{\mathrm{d}}(m)$, where the bias $\xi_{\mathrm{d}}=g \mu_{\mathrm{B}}\left|m-m^{\prime}\right| H_{\mathrm{d}, \mathrm{z}}$. As a result, thermally activated tunneling takes place predominantly via spin levels for which this ratio is not too far from unity [60].

The effect of dipolar interactions becomes even more dramatic at very low temperatures, when only the ground state doublet $( \pm S$ at $H=0)$ is populated. Since $\Delta_{S}$ is usually many orders of magnitude smaller than the 
typical $\xi_{\mathrm{d}}(S)$, only those molecules for which the local bias is either smaller than $\Delta_{S}$ or can be compensated by hyperfine interactions with magnetic nuclei are able to flip their spin $[31,67]$. Relaxation becomes then a purely collective process, because the tunneling of each spin changes the local fields acting on other crystal sites. The rate and time evolution depend on the symmetry of the lattice and also on whether the system is evolving towards a paramagnetic or a magnetically ordered state $[32,68,69]$.

Transverse dipolar field components $H_{\mathrm{d}, \mathrm{x}}$ and $H_{\mathrm{d}, \mathrm{y}}$ affect also spin tunneling and relaxation processes. Off-diagonal anisotropy terms in Eq.(1.3) are even. Therefore, they can only connect states $m$ and $m^{\prime}$ provided that $\left|m-m^{\prime}\right|$ is even too [70]. This condition applies at zero field, but not at some of the crossing fields defined by Eq. (1.14). In particular, tunneling would be strictly forbidden at crossing fields with odd "n". Transverse dipolar fields can contribute to break down such "selection rules", as they enable the quantum mixing between any pair of states [60]. This effect explains why magnetization steps (see Fig. 1.4) are observed at all crossing fields even in very precisely aligned crystals that are free from molecular disorder [71].

\subsubsection{Experimental determination of the average interaction fields}

The first of the effects described above provides a suitable method to measure the effective interaction field $H_{\text {eff, } \mathrm{z}}$ [cf Eq.(1.9)]. The method makes use of the strong sensitivity of quantum tunneling to the presence of even small bias magnetic fields. Tunneling resonances occur at well defined local magnetic fields $H_{z, \mathrm{n}}(m)$ given by Eq. (1.5). However, the magnetic bias field acting on each molecule in a crystal consists of the applied field $H_{z}$ plus contributions arising from the interactions with other molecules. The resonant field must therefore fulfill the following approximate condition

$$
H_{z, \mathrm{n}}(m)=H_{z}-\widetilde{N}_{z z} M_{z}+H_{\mathrm{eff}, \mathrm{z}}
$$

where $M_{z}=n g \mu_{\mathrm{B}}\left\langle S_{z}\right\rangle_{T}$ is the volumic longitudinal magnetization and $n$ is the concentration of spins per unit of volume. It can be expected that $H_{\mathrm{eff}, \mathrm{z}}$ depends on the spin configuration of a crystal, i.e. on $M_{z}$, thus the external field $H_{z}$ that fulfills condition (1.14) also does.

This dependence can be explored experimentally by sweeping the magnetic field back and forth across a given crossing field. Figure 1.4 shows the magnetization measured as this procedure is repeated near the first crossing field $(n=1)$ of $\mathrm{Mn}_{12}$ acetate, at $T=2.5 \mathrm{~K}$. The magnetization step, associated with this first tunneling resonance, shifts towards lower $H_{z}$ as $M$ increases. In fact, the dependence is close to linear, thus showing that the effective $H_{\text {eff, } z}$ is nearly proportional to $M_{z}$ too. Correcting from the demagnetization factor of the crystal, Eq. (1.14) gives $H_{\text {eff }, \mathrm{z}} \simeq \lambda M_{z}$, with $\lambda \equiv J_{\text {eff }} / n\left(g \mu_{\mathrm{B}}\right)^{2} \simeq 6$. For a magnetically polarized crystal of $\mathrm{Mn}_{12}$ acetate, with $M_{z}=M_{\mathrm{s}} \simeq 96 \mathrm{G}$, the maximum $H_{\text {eff }}$ amounts then to approximately 575 Oe. Taking into account 

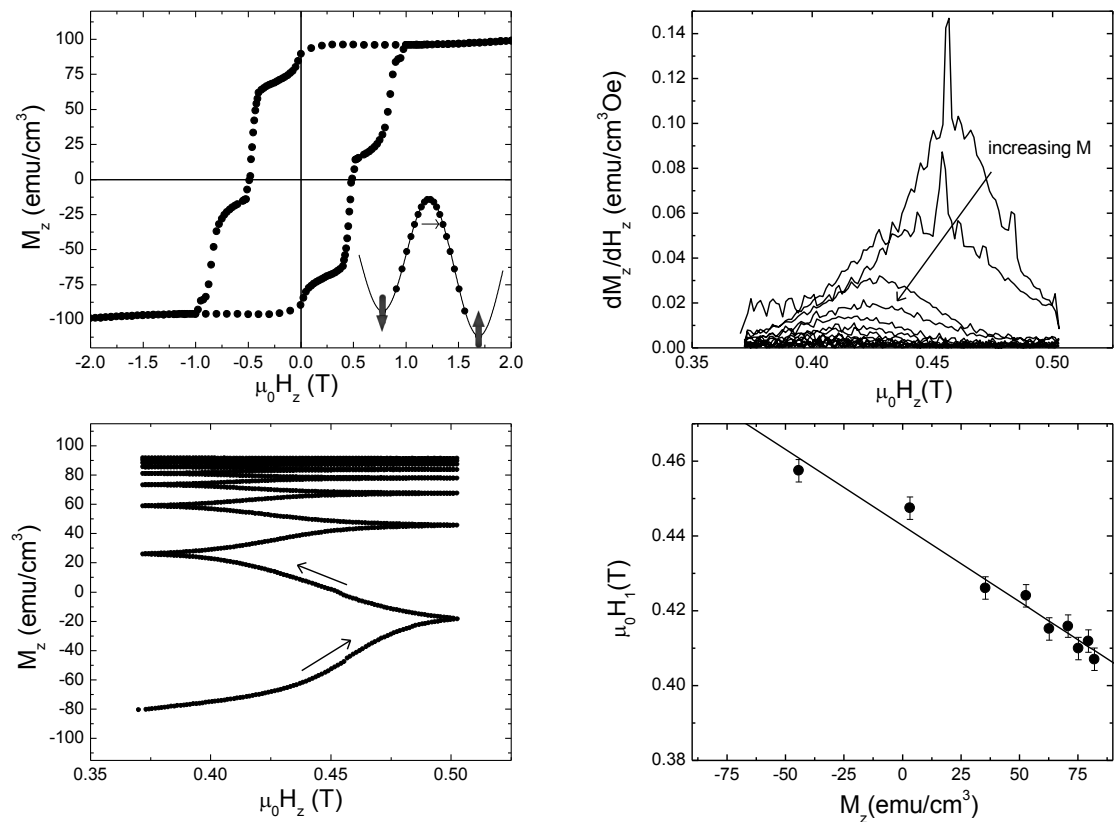

Fig. 1.4. A: Magnetization hysteresis loop of $\mathrm{Mn}_{12}$ acetate measured at $T=2.5 \mathrm{~K}$. The inset shows the structure of magnetic energy levels of this molecule at the first crossing field $H_{1}$, which corresponds to the magnetization step observed near $\mu_{0} H_{z}=$ $0.45 \mathrm{~T}$. B: Magnetization of $\mathrm{Mn}_{12}$ measured as the magnetic field is swept back and forth across this tunneling resonance. C: Magnetization derivative determined from these data. D: Position of $d M_{z} / d H_{z}$ maxima (resonant fields) as a function of magnetization

the experimental uncertainties involved (mainly associated with the accuracy in the determination of the demagnetization factor) this value agrees well with $H_{\text {eff }}=515 \pm 85$ Oe, reported in Ref. [72]. In the latter work, the hysteresis loop of fast relaxing $\mathrm{Mn}_{12}$ molecules [73] was used to monitor the magnetic field created by the standard, slower relaxing ones.

These results give also the opportunity to estimate the effective, or mean-field, interaction constant $J_{\text {eff }} / k_{\mathrm{B}} \simeq 7.5 \times 10^{-3} \mathrm{~K}$. For $\mathrm{Mn}_{12}$ clusters, $\Omega_{0} / k_{\mathrm{B}} \simeq 19 \mathrm{~K}$, thus it is much larger than $T_{\mathrm{c}}$. Mean-field Eqs. (1.10) and (1.12) are therefore applicable. The above value of $J_{\text {eff }}$ gives then rise to a critical temperature $T_{\mathrm{c}}=0.75 \mathrm{~K}$ for $\mathrm{Mn}_{12}$ acetate, close to the experimental $T_{\mathrm{c}} \sim 0.9 \mathrm{~K}$ (see section 1.5$)$. 


\subsection{Dipolar order of molecular nanomagnets with low magnetic anisotropy. Ferromagnetism in $\mathrm{Mn}_{6}$}

One of the simplest ways to obtain a dipolar magnet is to look for high-spin molecules having sufficiently weak magnetic anisotropy, thus also low energy barriers opposing the spin reversal. In this section, I briefly describe results of experiments performed on one of such molecules, $\mathrm{Mn}_{6} \mathrm{O}_{4} \mathrm{Br}_{4}\left(\mathrm{Et}_{2} \mathrm{dbm}\right)_{6}$, hereafter abbreviated as $\mathrm{Mn}_{6}[44,47,74]$.

The molecular core of $\mathrm{Mn}_{6}$, shown in the inset of Fig. 1.5, is a highly symmetric octahedron of $\mathrm{Mn}^{3+}$ ions (with spin $s=2$ ) that are ferromagnetically coupled via strong intra-cluster super-exchange interactions. Its ground magnetic state is a $S=12$ multiplet. The net magnetocrystalline anisotropy of this cluster proves to be very small, with $D \simeq 0.013 \mathrm{~K}[44,47]$. The classical energy barrier separating spin-up and spin-down states is then $U_{\mathrm{cl}} / k_{\mathrm{B}} \simeq 1.9$ $\mathrm{K}$, much smaller than $U_{\mathrm{cl}} / k_{\mathrm{B}} \simeq 70 \mathrm{~K}$ found for $\mathrm{Mn}_{12}$ clusters. $\mathrm{Mn}_{6}$ crystallizes in a monoclinic lattice with 4 molecules per unit cell [74] bound together only by Van der Waals forces. Inter-cluster super-exchange interactions are therefore expected to be negligible.

As a result of its weak magnetic anisotropy, the equilibrium magnetic susceptibility and specific heat of $\mathrm{Mn}_{6}$ can be measured down to very low temperatures. Curves measured for $H=0$ are shown in Fig. 1.5. Contributions associated with lattice vibrations and hyperfine interactions dominate $c$ measured above $2 \mathrm{~K}$ and below $100 \mathrm{mK}$, respectively. Between these two limits, $c$ is mainly due to the thermal population of molecular spin levels, split by the magnetic anisotropy and dipole-dipole interactions [cf Eq. (1.2)]. This magnetic contribution shows a sharp peak at 0.15(2) K. The magnetic entropy change, estimated from data measured between $0.08 \mathrm{~K}$ and $4 \mathrm{~K}$, amounts to $3.4 k_{B}$ per molecule, thus very close to the maximum entropy $\Delta S_{\mathrm{m}}=k_{\mathrm{B}} \ln (2 S+1)=3.22 k_{\mathrm{B}}$ of a $S=12$ spin multiplet. It therefore seems appropriate to assign the peak in $c$ to the onset of long-range magnetic order.

It is worth pointing out that the magnetic anisotropy of $\mathrm{Mn}_{6}$, despite its weakness, leaves its mark on the nature of the long-range order that arises below $T_{\mathrm{c}}$. The magnetic entropy change measured between $0.08 \mathrm{~K}$ and $T_{c}$ amounts to about $1 k_{\mathrm{B}}$ per spin, thus not far above $\Delta S_{\mathrm{m}}=k_{\mathrm{B}} \ln (2)=0.7 k_{\mathrm{B}}$ that is expected for an effective spin- $1 / 2$ system. This shows that, because of the low value of $T_{\mathrm{c}}$, mainly the lowest energy spin states (with $m= \pm 12$ ) take part in the magnetic ordering.

Information on the character of the magnetic order, i.e. whether it corresponds to a ferro- or antiferromagnetic phase, can be obtained from the ac magnetic susceptibility data shown on the right-hand side of Fig. 1.5. The real susceptibility component $\chi^{\prime}$ shows a sharp maximum at $T_{\mathrm{c}}=0.161(2) \mathrm{K}$, close to the ordering temperature estimated from heat capacity measurements. These data are compared with the paramagnetic susceptibility of $\mathrm{Mn}_{6}$, calculated by taking into account the effects of the magnetic anisotropy and of the sample's demagnetization factor. The experimental susceptibility lies clearly 

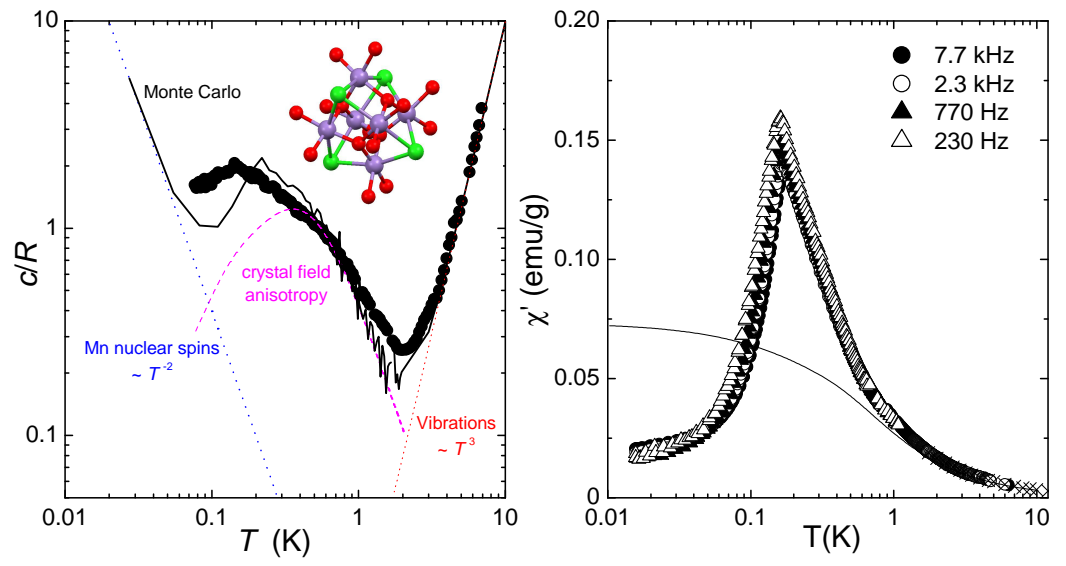

Fig. 1.5. Left: Dots, zero-field specific heat of $\mathrm{Mn}_{6}$; dotted line, phonon contribution; dashed line: Schottky contribution due to crystal field splitting of the $\mathrm{S}=12$ multiplet as calculated with Eq.(1.3) for $D / k_{\mathrm{B}}=0.013 \mathrm{~K}$; dotted curve: nuclear contribution expected from the ${ }^{55} \mathrm{Mn}$ nuclear spins. Solid line: equilibrium specific heat derived from Monte Carlo calculations, and including all previous contributions as well as the effects of dipole-dipole interactions. The inset shows a sketch of the symmetric octahedral core of each $\mathrm{Mn}_{6}$ molecule, with total spin $S=12$. Right: Real component of the ac susceptibility of $\mathrm{Mn}_{6}$ measured at several frequencies. The solid line gives the paramagnetic susceptibility of non interacting $\mathrm{Mn}_{6}$ clusters. These calculations include the effects of the zero-field splitting and of demagnetizing fields

above this prediction, thus suggesting that the magnetic order in $\mathrm{Mn}_{6}$ is ferromagnetic, i.e., that $\theta>0$ in Eq. (1.10). Figure 1.6 shows indeed that, above $T_{\mathrm{c}}$, the intrinsic magnetic susceptibility $\chi_{\mathrm{i}}$, corrected for demagnetization effects, follows accurately Curie-Weiss law, with $C=0.034(1) \mathrm{emuK} / \mathrm{g}$ Oe and $\theta=0.20(3) \mathrm{K}$. These data agree with the fact that three-dimensional dipolar lattices must be close approximations of mean-field models. The ferromagnetic nature of the ordered phase is also confirmed by the fact that relatively weak magnetic fields completely suppress the heat capacity maximum [44, 47].

The dipolar magnetic order in $\mathrm{Mn}_{6}$ has been investigated by means of Monte Carlo simulations, which are described in detail in refs. [44, 47]. As it has been have argued in section 1.2 , because $U_{\mathrm{cl}} / k_{\mathrm{B}} \gg T_{\mathrm{c}}$ only states with $m= \pm 12$ are appreciably populated at and below $T_{\mathrm{c}}$. This justifies the use of the Ising Hamiltonian (1.6) to describe the magnetic ordering of $\mathrm{Mn}_{6}$ molecular nanomagnets.

Monte Carlo simulations show that the ground state is ferromagnetically ordered, as observed, and predict a shape for $c$ that is in reasonably good good agreement with the experiment. The solid line in Fig. 1.5 shows $c$ cal- 


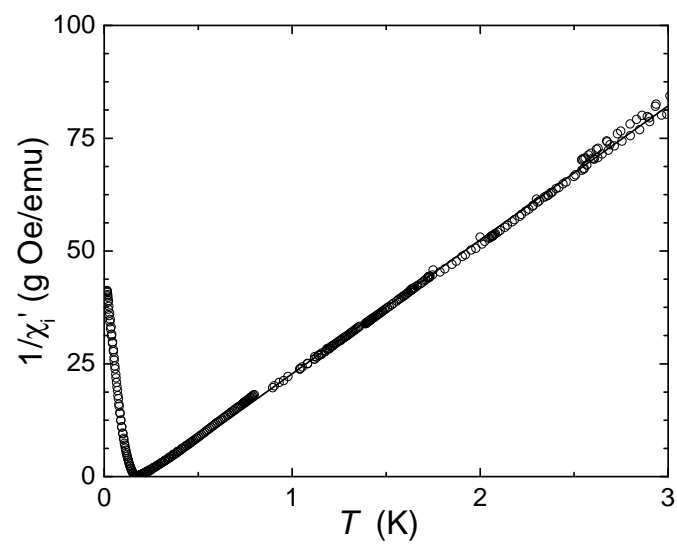

Fig. 1.6. Reciprocal magnetic susceptibility of $\mathrm{Mn}_{6}$ corrected for demagnetization effects. The solid line is a least-squares fit of a Curie-Weiss law to the data measured above $0.3 \mathrm{~K}$

culated assuming that all molecular anisotropy axes $(z)$ point along one of the two nearly equivalent short axes of the actual lattice. Similar results were obtained for other orientations of the anisotropy axes. These simulations give $T_{c}=0.22 \mathrm{~K}$, which is slightly above the experimental $T_{\mathrm{c}}=0.161(2) \mathrm{K}$. As it was argued in [47], this difference can be assigned to the finite value of the anisotropy. Model calculations, performed for the same crystal structure but assuming classical Heisenberg spins with varying anisotropy show that different ferromagnetic ground states are possible, depending on the competition between local crystal field effects and long-range dipolar interactions. The strong dependence of variation of $T_{\mathrm{c}}$ on the sign and orientation of the magnetic anisotropy, as well as the form of the calculated and observed specific heat anomalies turn out to be specific for dipolar interactions, and differ widely from the analogues for usual ferromagnets, coupled via super-exchange interactions [51].

Spin dynamics close to $T_{\mathrm{c}}$

Ac susceptibility data provide also interesting information on the dynamics of spins close to and below $T_{\mathrm{c}}$. The maximum value of $\chi^{\prime}$ is seen to weakly vary with the frequency $\omega / 2 \pi$ of the ac excitation magnetic field. This variation suggests that, for the highest frequencies employed in these experiments, spins begin to deviate from equilibrium already above $T_{\mathrm{c}}$. A more dramatic effect is observed below the ordering temperature. The real susceptibility component $\chi^{\prime}$ decreases rapidly, thus suggesting that the ferromagnetic response is also being blocked by slow relaxation processes.

These phenomena can be understood, at least qualitatively, if one takes into account once more the finite magnetic anisotropy of $\mathrm{Mn}_{6}$ clusters. The 
superparamagnetic blocking of $\mathrm{Mn}_{6}$ spins is expected to occur at $T_{b} \simeq$ $D S^{2} / k_{\mathrm{B}} \ln \left(1 / \omega \tau_{0}\right)$. Setting $\tau_{0}=10^{-8} \mathrm{~s}$, which is a typical value found for other SMMs, gives $T_{\mathrm{b}} \simeq 0.25 \mathrm{~K}$ for $\omega / 2 \pi=7.7 \mathrm{kHz}$. In other words, for $T \rightarrow T_{\mathrm{c}}$, the approach to equilibrium begins to be hindered by the anisotropy barrier of each molecular spin. These estimates have been confirmed by recent experiments performed on different derivatives of $\mathrm{Mn}_{6}$, which show slightly lower values of $T_{\mathrm{c}}[75]$. In these samples, a frequency-dependent super-paramagnetic blocking is observed below $0.2 \mathrm{~K}$.

Below $T_{\mathrm{c}}$, the slow magnetic relaxation contributes to "pin" magnetic domain walls. This effect accounts for the sharp decrease observed in the linear magnetic susceptibility. The dynamics associated with the displacement of domain walls in Ising-like dipolar ferromagnets had not been simulated until recently [76]. The present experiments suggest that, in the case of $\mathrm{Mn}_{6}$, the magnetization dynamics close to $T_{\mathrm{c}}$ seems to be dominated by thermal fluctuations. An interesting question that needs to be addressed by future experimental work is whether domain walls move by flipping one molecular spin at a time or via a collective process.

\subsection{Dipolar order in a transverse magnetic field. Ferromagnetism in $\mathrm{Mn}_{12}$ acetate}

\subsubsection{Magnetic ordering via pure quantum tunneling}

For many of the best known SMMs, magnetic anisotropy barriers are so high that, close to $T_{\mathrm{c}}$, thermally activated spin flips take place in time scales that are much longer than the typical experimental time scales. Under these conditions, only pure spin tunneling events contribute to the magnetization dynamics. The precise mechanism by which quantum tunneling enables the spin system to exchange energy with the lattice is not yet fully understood. However, in spite of their intrinsically quantum nature and the fact that they are independent of temperature, experiments show that these quantum fluctuations are nevertheless able to bring the spin system to equilibrium with the thermal bath $[45,64,77,78]$. These processes enable also the onset of longrange magnetic order in crystals of SMMs. However, as it has been mentioned above, they are also rather slow, with time scales of the order of many hours for $\mathrm{Fe}_{8}$ or even months, as it is the case for $\mathrm{Mn}_{12}$ clusters.

Quantum tunneling can be, to some extent, controlled by chemical design. The symmetry of the cluster magnetic cores determines the structure of the spin Hamiltonian (1.3). Lowering the molecular symmetry allows the presence of lower order off-diagonal terms, which contribute to enhance quantum tunneling probabilities. In clusters with a $\mathrm{Mn}_{4} \mathrm{O}_{3} \mathrm{X}$ cubane magnetic core, this effect has been induced via the chemical binding to different ligands $\mathrm{X}$. Then, while highly symmetric $\mathrm{Mn}_{4} \mathrm{O}_{3} \mathrm{Cl}$ and $\mathrm{Mn}_{4} \mathrm{O}_{3} \mathrm{OAc}$ clusters [79] show the typical SMM behaviour, with blocking temperatures in the vicinity of $1 \mathrm{~K}$, the 
spins of a strongly distorted $\mathrm{Mn}_{4} \mathrm{O}_{3} \mathrm{MeOAc}$ [80] remain in equilibrium down to very low temperatures. In the latter sample, the heat capacity shows the onset of long-range magnetic order at $T_{\mathrm{c}}=0.2 \mathrm{~K}$ [45]. This value is found to be larger than the maximum critical temperature compatible with dipolar interactions. Therefore, in this case super-exchange interactions probably play a non-negligible role. This example shows that conclusions on the existence of pure dipolar order cannot be drawn from qualitative arguments alone, and that a quantitative comparison with theoretical predictions are always necessary. Ferromagnetic order has also been observed in crystals of low symmetry $\mathrm{Ni}_{4}$ clusters, which show one of the highest tunneling rates (of order $10^{5} \mathrm{~s}^{-1}$ ) ever measured $[48,75]$.

\subsubsection{Quantum annealing}

An additional trick, based on the above considerations, can be played in crystals of SMMs having their magnetic anisotropy axes aligned along given crystallographic directions. Transverse components of the Zeeman interaction (1.4), i.e. those associated with magnetic field components $H_{x}$ and $H_{y}$, also induce quantum tunneling of the spins. Since off-diagonal terms play, to some extent, a role comparable to that of a kinetic energy in the tunneling of a material particle, the magnetic field enables then to "tune" the effective tunneling mass. This ability has been used to directly detect the existence of a quantum tunnel splitting $[38,39]$ and to induce quantum interference phenomena between different tunneling trajectories [37]. Naturally, it can also be applied to explore the existence of a magnetically ordered phase.

The basic protocol for this "quantum annealing" (see also $[81,82]$ ) is shown in Fig. 1.7. By increasing the transverse magnetic field $H_{\perp}$, tunneling probabilities are rapidly enhanced, thus at some point spins are able to reach thermal equilibrium with the lattice. If at this temperature and field the spin system remains ferromagnetically ordered, a net magnetization will be recorded that will "freeze" as the magnetic field is set back to zero through the irreversibility field $H_{\text {irr }}$. The latter field, thus also the result of the quantum annealing process, depend on the experimental probe and its characteristic time scales. This dependence is shown in 1.7 that compares data derived for $\mathrm{Mn}_{12}$ acetate using heat capacity [83] and magnetic neutron diffraction experiments [46].

\subsubsection{The quantum Ising model}

The control of quantum tunneling fluctuations by an external magnetic field offers an additional and very attractive possibility for fundamental physical studies. As it has been discussed in section 1.2, dipole-dipole interactions between highly anisotropic spins (with $D \rightarrow \infty$ ) can be approximated by a spin-1/2 Ising Hamiltonian. In the presence of a transverse magnetic field, a crystal of perfectly oriented SMMs can therefore provide a material realization 


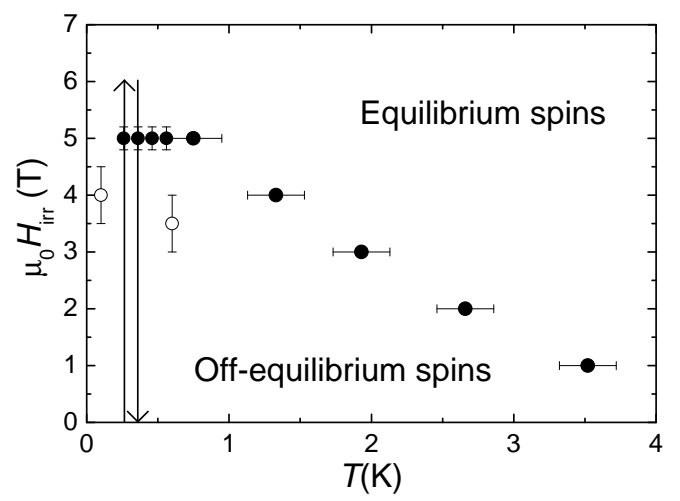

Fig. 1.7. Irreversibility transverse magnetic field separating equilibrium and nonequilibrium conditions of $\mathrm{Mn}_{12}$ acetate spins. Solid and open dots have been determined from specific heat data (experimental time constant $\sim 1 \mathrm{~s}$ ) and magnetic neutron diffraction experiments (experimental time constant $\sim 10^{4} \mathrm{~s}$ ), respectively. The arrows show schematically the quantum annealing protocol employed to explore the existence of long-range magnetic order at very low temperatures

of the quantum Ising model [30]. The spin Hamiltonian of this model reads as follows

$$
\mathcal{H}=-\frac{S^{2}}{2} \sum_{i} \sum_{j \neq i} J_{i j} \sigma_{\mathrm{i}, \mathrm{z}} \sigma_{\mathrm{j}, \mathrm{z}}-\Delta \sum_{i} \sigma_{\mathrm{i}, \mathrm{x}}
$$

where $J_{i j}$ are longitudinal couplings (here of dipolar origin) and $\Delta$ is the ground-state tunnel splitting which depends on and vanishes with $H_{\perp}$. Equation (1.15) represents the archetypical (and arguably the simplest) model for a quantum phase transition $[40,41]$. The classical long-range order that exists for $H_{\perp}=\Delta=0$ (ferromagnetic or anti-ferromagnetic) competes with field-induced quantum fluctuations. The magnetic phase diagram, representing $\left(T_{\mathrm{c}}, \Delta_{\mathrm{c}}\right)$ [or, equivalently, $\left.\left(T_{\mathrm{c}}, H_{\mathrm{c}}\right)\right]$ points at which magnetic order is suppressed, can be calculated using the mean-field approximation (1.11). The magnetic phase boundary between the ordered and paramagnetic phases is defined by the following equation

$$
\frac{k_{\mathrm{B}} T_{\mathrm{c}}\left(H_{\perp}=0\right)}{\Delta_{\mathrm{c}}}=\operatorname{coth}\left(\frac{\Delta_{\mathrm{c}}}{k_{\mathrm{B}} T_{\mathrm{c}}}\right)
$$

where $T_{\mathrm{c}}\left(H_{\perp}=0\right)=\theta$ is given by Eq. (1.12). At $T=0$, magnetic order is completely destroyed at $\Delta_{\mathrm{c}}=k_{\mathrm{B}} T_{\mathrm{c}}\left(H_{\perp}=0\right)$.

Quantum phase transitions have been extensively studied in recent years. Examples include the superconductor insulator transition in cuprates [84-86], 
the onset of antiferromagnetism in heavy fermions [87], the pressure driven insulator-metal transition in $\mathrm{V}_{2} \mathrm{O}_{3}$ [88], and the magnetic transitions driven by field $\left(\mathrm{LiHoYF}_{4}[89]\right)$ or concentration $\left(\mathrm{Cr}_{x} \mathrm{~V}_{1-x}\right.$ alloys [90]). In addition to their intrinsic interest, a plethora of new properties arise at nonzero temperature.

In spite of this intense activity, pure realizations of the quantum Ising model with magnetic materials are very scarce. As it happens with dipolar magnetism in general, lanthanide-based insulators seem to be a natural choice for these studies $[89,91]$. However, the strong hyperfine interactions seriously limit the observation of the intrinsic quantum criticality in these materials $[23,24]$. Crystals of single molecule magnets, for which hyperfine interactions are typically much weaker, are then very attractive candidates.

\subsubsection{Magnetic order in $\mathrm{Mn}_{12}$ acetate}

\section{Neutron diffraction experiments}

The cluster of $\mathrm{Mn}_{12}$ acetate [92], the first and most extensively studied member of the family of single-molecule magnets, is shown in Fig. 1.2. It contains 12 manganese atoms linked via oxygen atoms, with a sharply-defined and monodisperse size. At low temperatures, each of them exhibits slow magnetic relaxation and hysteresis, due to the combination of an $S=10$ magnetic ground state with appreciable uniaxial magnetic anisotropy. Finally, they organize to form tetragonal molecular crystals. Monte Carlo simulations [7], as well as mean field calculations [76], predict that $\mathrm{Mn}_{12}$ acetate must order ferromagnetically as a result of dipolar interactions between molecular spins. The critical temperatures derived from these calculations are $T_{\mathrm{c}}=0.5 \mathrm{~K}$ and $0.8 \mathrm{~K}$, respectively. Therefore, these crystals seem to offer a nearly perfect realization of the quantum Ising model (1.15). To which extent this is indeed the case will be discussed in the following.

Not surprisingly, detecting the presence of long-range magnetic order in $\mathrm{Mn}_{12}$ faces some important experimental difficulties. Spin reversal via resonant quantum tunneling $[33-35,63]$ becomes extremely slow at low temperatures (of order two months at $T=2 \mathrm{~K}$ ). For the time scales $\sim 10^{2}-10^{4} \mathrm{~s}$ of a typical experiment $T_{\mathrm{b}} \sim 3 \mathrm{~K}$, thus much higher than the ordering temperature $T_{\mathrm{c}}$. Equilibrium conditions can be explored via the application of the quantum annealing protocol described above. Magnetic diffraction of thermal neutrons is a suitable tool for these studies because it can probe different components of the magnetization vector, in particular $M_{z}$ [93], in the presence of a transverse magnetic field. In addition to this, diffraction patterns provide a very accurate determination of the crystal's orientation. And finally, the typical data acquisition times required to obtain reasonably good results are very long, which gives rise to smaller values of $H_{\text {irr }}$ (see Fig. 1.7). In the experiments whose results are described below [46], a $\sim 0.5 \times 0.5 \times 1.5 \mathrm{~mm}^{3}$ 

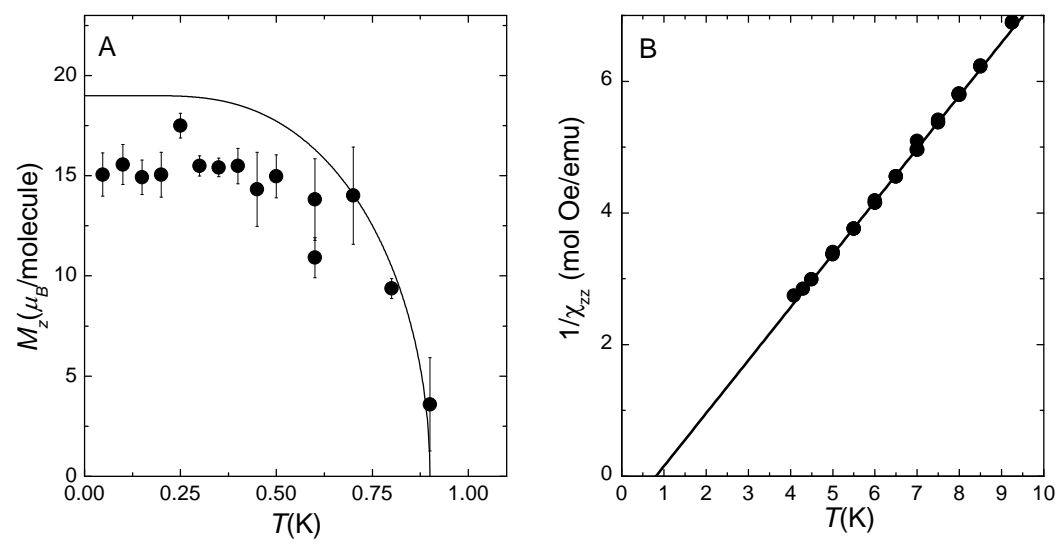

Fig. 1.8. Left: Longitudinal magnetization $M_{z}$ of $\mathrm{Mn}_{12}$ acetate obtained from neutron diffraction data measured at $\mu_{0} H_{\perp}=0$ after decreasing the transverse magnetic field from $6 \mathrm{~T}$ at each temperature. Solid lines are calculations (for a perfect orientation of the magnetic field perpendicular to the easy axes of all molecules) that include interactions via the mean-field Hamiltonian (1.7). Right: Reciprocal parallel susceptibility measured at $T>4.5 \mathrm{~K}$ (i.e. above $T_{\mathrm{b}}$ ) along the $\boldsymbol{c}$ crystallographic axis. The solid line is a least-squares linear fit, giving $\theta=0.8(1) \mathrm{K}$

single crystal of deuterated $\mathrm{Mn}_{12}$ acetate was attached to the mixing chamber of a ${ }^{3} \mathrm{He}-\mathrm{He}^{4}$ dilution refrigerator with its $\boldsymbol{c}$ axis perpendicular (up to a maximum deviation of about 0.1(1) degrees) to the magnetic field.

Given the strong magnetic anisotropy of $\mathrm{Mn}_{12}$ clusters, the magnetization is confined in the plane defined by $\boldsymbol{c}$ and $\boldsymbol{H}$, with components $M_{z}$ and $M_{\perp}$, respectively. At $4 \mathrm{~K}$, that is, in the paramagnetic state, $M_{z}=0$ and $M_{\perp}$ is proportional to $H_{\perp}$. For $T \leq 900 \mathrm{mK}$, by contrast, a large additional contribution to the magnetic diffraction intensities shows up for $\mu_{0} H_{\perp}<5 \mathrm{~T}$, but only provided that $H_{\perp}$ is first raised above $H_{\text {irr }}$ at each temperature. As shown in Fig. 1.8 , this contribution reflects the onset of a non-zero spontaneous $M_{z}$ below $T_{\mathrm{c}}=0.9(1) \mathrm{K}$. The latter value is close to the Weiss temperature $\theta \simeq 0.8(1) \mathrm{K}$ extracted from the extrapolation of $1 / \chi_{z z}^{\prime}$ data measured above $T_{\mathrm{b}}$ (Fig. 1.8). These data suggest that $\mathrm{Mn}_{12}$ acetate does indeed order ferromagnetically, as predicted. The experimental $T_{\mathrm{c}}$ is in good agreement with mean-field calculations [76]. However, it is nearly a factor two larger than the critical temperature derived from Monte Carlo calculations for pure dipolar interactions [7]. Therefore, the presence of weak super-exchange interactions contributing to enhance the magnetic ordering temperature of $\mathrm{Mn}_{12}$ acetate cannot be completely ruled-out. The same conclusion was derived from the analysis of the susceptibilities and Weiss temperatures of different $\mathrm{Mn}_{12}$ derivatives [54]. 

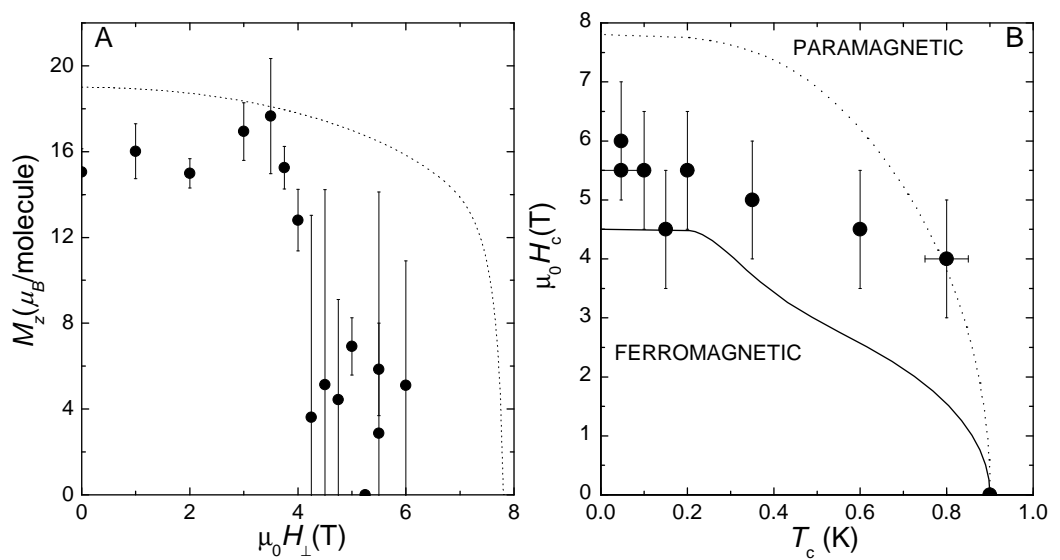

Fig. 1.9. Left: Longitudinal magnetization $M_{z}$ of $\mathrm{Mn}_{12}$ acetate measured while decreasing the transverse magnetic field $\mu_{0} H_{\perp}$ from $6 \mathrm{~T}$ at $T=47 \mathrm{mK}$. The solid line has been calculated using Eq. (1.7) and the parameters given in the text. Right: Magnetic phase diagram of $\mathrm{Mn}_{12}$ acetate. The dotted line was obtained using the mean-field Hamiltonian (1.7) for perfectly aligned anisotropy axes. The solid line is the mean field prediction following from the random-field Hamiltonian (1.18), which includes effects of molecular disorder [50]

Another remarkable finding, shown in Fig. 1.9, is the strong dependence of $M_{z}$ on $H_{\perp}$. At the minimum temperature $T=47 \mathrm{mK}, M_{z}$ is approximately zero for $\mu_{0} H_{\perp}>5.5(5) \mathrm{T}$ and then it increases when decreasing $\mu_{0} H_{\perp}$, reaching $16 \mu_{\mathrm{B}}$ per molecule at zero field. These results show that a transverse magnetic field tends to suppress the ferromagnetic order. The $T_{\mathrm{c}}-H_{\mathrm{c}}$ magnetic phase diagram of $\mathrm{Mn}_{12}$ acetate is shown on the right-hand panel of Fig. 1.9. A ferromagnetic phase exists for sufficiently low temperatures and transverse magnetic fields. The qualitative resemblance between the effects caused by temperature and field is typical of systems undergoing a quantum phase transition. However, as it is argued in the following, understanding the true nature of the field-dependent transition can only be achieved by a quantitative comparison to theoretical simulations.

\section{Comparison to the quantum Ising model predictions}

For a perfectly oriented crystal of $\mathrm{Mn}_{12}$ molecules in a transverse magnetic field, the mean-field Hamiltonian (1.7) can be written as

$$
\begin{aligned}
\mathcal{H}= & -D S_{z}^{2}+B S_{z}^{4}+\frac{C}{2}\left(S_{+}^{4}+S_{-}^{4}\right) \\
& -g \mu_{\mathrm{B}} H_{\perp}\left(S_{x} \cos \phi+S_{y} \sin \phi\right)-J_{\mathrm{eff}}\left\langle S_{z}\right\rangle S_{z}
\end{aligned}
$$


Spectroscopic measurements [94-99] give $g=1.9, D / k_{\mathrm{B}}=0.6 \mathrm{~K}, B / k_{\mathrm{B}}=$ $-10^{-3} \mathrm{~K}$, and $C / k_{\mathrm{B}}=-6.1 \times 10^{-5} \mathrm{~K}$. Experiments performed on single crystals [98] provide also the orientation of the fourth-order anisotropy axes $x$ and $y$ with respect to the crystallographic axes $\boldsymbol{a}$ and $\boldsymbol{b}$. In the neutron diffraction experiments, $\boldsymbol{H}$ was approximately parallel to the $1 \overline{1} 0$ crystallographic direction, which corresponds to $\phi \simeq \pi / 4$. The mean-field constant $J_{\text {eff }}$ was set to $9 \times 10^{-3} k_{\mathrm{B}}$ that, according to Eq. (1.12), fits the experimental $T_{\mathrm{c}}=0.9$ $\mathrm{K}$. The above $J_{\text {eff }}$ value is close to $7.5 \times 10^{-3} k_{\mathrm{B}}$ determined from quantum tunneling experiments described in section 1.3.3.

Predictions for $M_{z}$ as a function of temperature and magnetic field that follow from Eq. (1.17) are shown in Figs. 1.8 and 1.9(A). These calculations account reasonably well for the temperature dependence of $M_{z}$ measured at $H_{\perp}=0$. The fact that $M_{z}$ remains smaller than the saturation magnetization of $19 \mu_{\mathrm{B}}$ per molecule even at $T \rightarrow 0$ can be ascribed to non equilibrium effects. It probably arises from reversed spins that remain frozen as the magnetic field is reduced below $\mu_{0} H_{\mathrm{irr}} \simeq 4 \mathrm{~T}$, because quantum tunneling rates become then extremely slow. However, the same model fails to account for the field-dependent behavior. In particular, the zero-temperature critical field $\mu_{0} H_{\mathrm{c}}(T=0)$, at which quantum fluctuations finally destroy the long-range ferromagnetic order, is close to $8 \mathrm{~T}$, thus considerably higher than the experimental $\mu_{0} H_{\mathrm{c}} \simeq 5.5 \mathrm{~T}$. The discrepancy manifests itself also in the shape of the magnetic phase diagram at low temperatures, shown in Fig. 1.9(B).

Molecular disorder: random-field magnetism in $\mathrm{Mn}_{12}$ acetate

In the original analysis of the neutron diffraction experiments [46], the fielddependent magnetization was fitted by introducing a large and positive fourthorder off-diagonal parameter $C$, which "helps" the magnetic field in generating sufficiently strong quantum fluctuations. Disorder in the orientation of acetic acid solvent molecules can lower the local symmetry of $\mathrm{Mn}_{12}$ molecules and give rise, for some of them, to additional off-diagonal terms, such as $E\left(S_{x}^{2}-\right.$ $S_{y}^{2}$ ), not allowed for the ideal molecular symmetry [100]. The presence of such terms has been put into evidence by magnetic relaxation $[101,102]$ and spectroscopic experiments [98], which are described elsewhere in this book. However, introducing such terms in Eq. (1.17) cannot, by itself, account for either the value of $H_{\mathrm{c}}$ or the magnetic-field diagram that are experimentally observed.

Molecular disorder can, however, affect ferromagnetism in a different, subtle manner. Some of the different isomers, associated with given orientations of the interstitial molecules with respect to $\mathrm{Mn}_{12}$ cores, have their easy axes $z$ tilted with respect to the crystallographic $c$ axis [100]. The tilting angles $\delta$ have been estimated by several experimental methods and turn out to be rather small, of the order of 1 deg., or even less [98, 103]. At zero field, it is therefore expected that their influence on the ferromagnetic order be small. However, their presence makes itself felt when a magnetic field is applied perpendicular to $\boldsymbol{c}$. As it was first pointed out by Millis and co-workers [10], some 
molecular sites then "see" a nonzero bias field $H_{z}$, which for $H_{\perp} \geq 3$ become already stronger than the maximum $H_{\text {eff }}$ associated with intermolecular magnetic interactions. Furthermore, the bias is randomly distributed among the different sites.

In order to describe these effects, the mean-field Hamiltonian for each molecule at site $\boldsymbol{r}_{\boldsymbol{i}}$ must include an additional random-field term [10,50]

$$
\begin{aligned}
\mathcal{H}\left(\boldsymbol{r}_{\boldsymbol{i}}\right)= & -D S_{z}^{2}+B S_{z}^{4}+\frac{C}{2}\left(S_{+}^{4}+S_{-}^{4}\right)-g \mu_{\mathrm{B}} H_{\perp}\left(\boldsymbol{r}_{\boldsymbol{i}}\right)\left(S_{x} \cos \phi+S_{y} \sin \phi\right) \\
& -g \mu_{\mathrm{B}} H_{z}\left(\boldsymbol{r}_{\boldsymbol{i}}\right) S_{z}-J_{\mathrm{eff}}\left\langle S_{z}\right\rangle S_{z}
\end{aligned}
$$

As can be expected, the effect of disorder is to suppress magnetic order for applied magnetic field values $H$ that are significantly smaller than the critical field of the pure quantum Ising model (1.7). The solid line in the right-hand panel of Fig. 1.9 shows the magnetic phase diagram derived [50] from Eq. (1.18), using the distribution of random easy axes tilts calculated by Park and co-workers [104]. In [50], it was shown that this model gives a fair account of the Weiss temperatures determined from the extrapolation of the reciprocal susceptibility (see Fig. 1.8), although its predictions tend to slightly overestimate $H_{\mathrm{c}}$ at any temperature. It can be seen that it also provides a better description of the low- $T /$ high- $H_{\perp}$ behavior obtained from magnetic neutron diffraction experiments. However, the discrepancy between experimental and theoretical values of $H_{\mathrm{c}}$ is even larger than that derived from magnetic data [50]. This suggests that the degree of interstitial disorder can be different for different crystals of $\mathrm{Mn}_{12}$ acetate and that these differences manifest themselves in the magnetic phase diagram.

These results illustrate the rich physical behavior of $\mathrm{Mn}_{12}$ acetate in the presence of a transverse magnetic field. This system provides a unique opportunity to investigate the interplay between dipolar interactions and randomness and represents one of the best material realizations of the random-field Ising model known to date.

\subsection{Magnetic order and quantum phase transition in $\mathrm{Fe}_{8}$}

The previous section illustrates some of the difficulties met in the search of pure quantum phase transitions with SMMs: molecular disorder turns $\mathrm{Mn}_{12}$ acetate into a realization of the classical random-field Ising model. In this section, I review experimental work performed on a crystal of $\mathrm{Fe}_{8}$ SMMs [49]. This molecular material [105] possesses some properties that make it especially well suited for these studies, viz (i) classical Monte Carlo (MC) simulations suggest a ferromagnetic ground state with $T_{\mathrm{c}}=0.54 \mathrm{~K}$ [7] (ii) hyperfine interactions are much smaller than both the magnetic anisotropy and dipolar interactions, thus they cannot perturb quantum dynamics of SMMs and (iii) disorder is weak enough to avoid sizable random fields. 

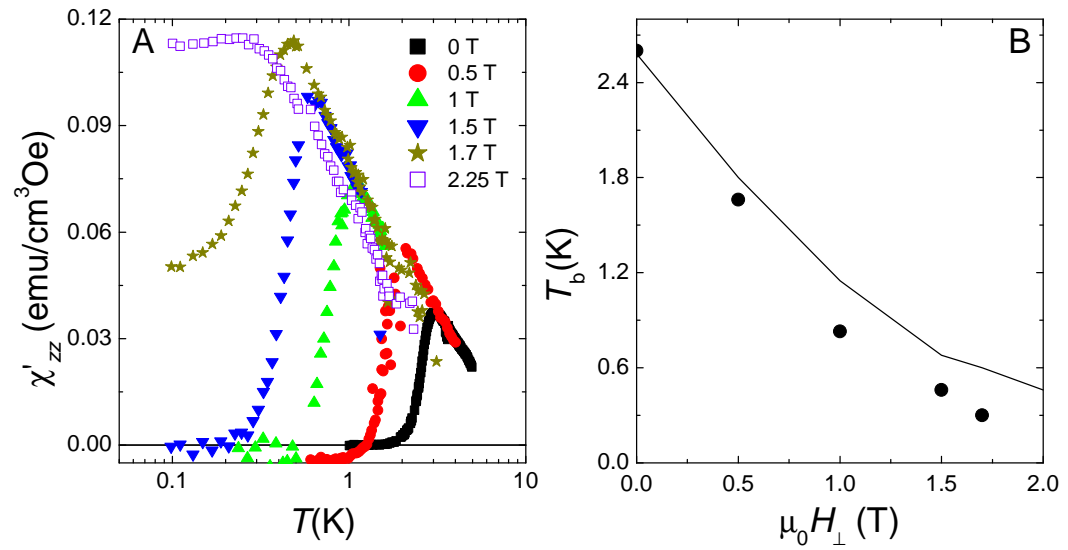

Fig. 1.10. A: Longitudinal in-phase ac susceptibility of $\mathrm{Fe}_{8}$ measured at $\omega / 2 \pi=333$ $\mathrm{Hz}$ and for different values of the transverse magnetic field $H_{\perp}$. B: Shift of the superparamagnetic blocking temperatures with increasing $H_{\perp}$. The solid line shows theoretical predictions for quantum spin-phonon relaxation that follow from Pauli's master equation as described in [60].

Each $\mathrm{Fe}_{8}$ molecule (brief for $\left[\left(\mathrm{C}_{6} \mathrm{H}_{15} \mathrm{~N}_{3}\right)_{6} \mathrm{Fe}_{8} \mathrm{O}_{2}(\mathrm{OH})_{12}\right]$ ) has a spin $S=$ 10 and a strong uniaxial magnetic anisotropy [106]. It can be described by Hamiltonian (1.3) with $D / k_{\mathrm{B}}=0.294 \mathrm{~K}, E / k_{\mathrm{B}}=0.046 \mathrm{~K}$, and $g=2[106$, 107]. $x, y$ and $z$ correspond to the hard, medium and easy magnetization axes that, in the triclinic crystal structure of $\mathrm{Fe}_{8}$, are common to all molecules [108].

Ac magnetic susceptibility experiments, reported in [49], were performed down to $90 \mathrm{mK}$ on a $1.6 \mathrm{mg}$ single crystal of approximate dimensions $1 \times 2 \times 1$ $\mathrm{mm}^{3}$. The magnetic easy axis $z$ was oriented approximately parallel to the ac excitation magnetic field. Therefore, these experiments give access to the longitudinal linear magnetic response that is expected to diverge close to a magnetic phase transition. The dc magnetic field was then carefully aligned with respect to the crystal axes with the help of a $9 \mathrm{~T} \times 1 \mathrm{~T} \times 1 \mathrm{~T}$ superconducting vector magnet, using the strong dependence of the paramagnetic $\chi_{z z}^{\prime}$ on the magnetic field orientation [49]. It was found that $\vec{H}$ is perpendicular $\left( \pm 0.05^{\circ}\right)$ to $z$ and close $\left(\phi \simeq 68^{\circ}\right)$ to the medium $y$ axis.

As expected for a high-anisotropy SMM, the ac susceptibility (Figure 1.10) of $\mathrm{Fe}_{8}$ deviates from equilibrium for low $H_{\perp}$ and low $T$, as shown by the vanishing of $\chi_{z z}^{\prime}$. The superparamagnetic blocking temperature $T_{\mathrm{b}}$ strongly depends on frequency. However, even for the lowest available frequencies, $T_{\mathrm{b}}$ remains much higher than $1 \mathrm{~K}$, thus also higher than the expected critical temperature. The same applies to heat capacity experiments that have been discussed in section 1.3.1 (cf Fig. 1.3). 

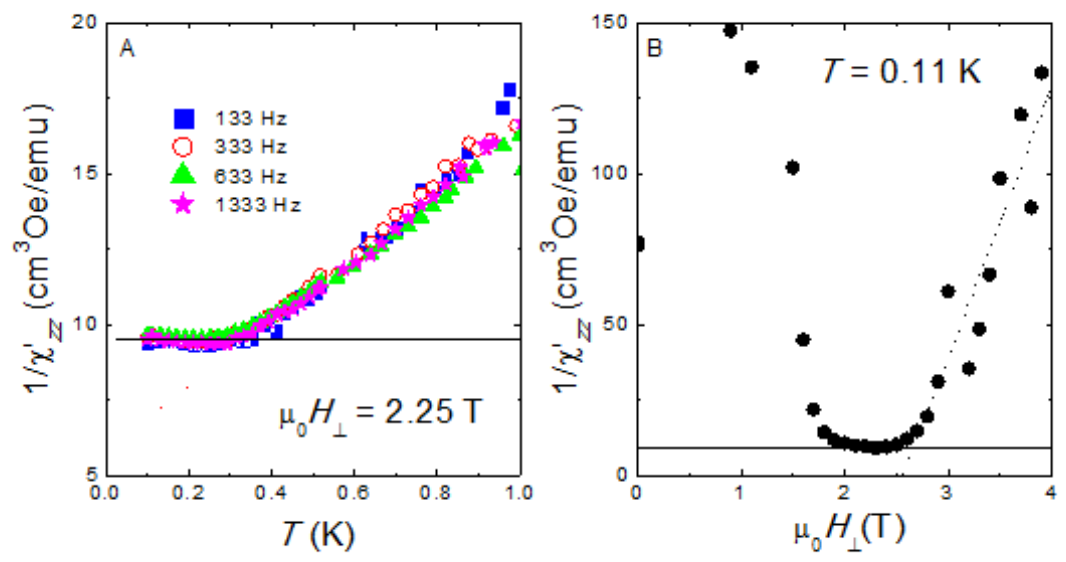

Fig. 1.11. Reciprocal in-phase ac susceptibility measured at $\mu_{0} H_{\perp}=2.25 \mathrm{~T}$ as a function of $T$ (left) and at $T=0.110 \mathrm{~K}$ as a function of $H_{\perp}$ (right). The crossovers between the "Curie-Weiss" law, observed at either high $T$ or high $H_{\perp}$ (dotted blue lines), and the ferromagnetic limit $1 / \chi_{\max }^{\prime}=\widetilde{N}_{z z}$ (solid red lines) give $T_{\mathrm{c}}$ $(=0.34(1) \mathrm{K})$ and $\mu_{0} H_{\mathrm{c}}(=2.65(5) \mathrm{T})$, respectively

As with $\mathrm{Mn}_{12}$ acetate, this situation can be reversed by enhancing quantum spin fluctuations via the application of $H_{\perp}$. As shown in Fig. 1.10, increasing $H_{\perp}$ reduces $T_{\mathrm{b}}$, thus showing that spins are able to attain thermal equilibrium at progressively lower temperatures. It is interesting to mention also that, besides enhancing the spin dynamics, the magnetic field also lowers the paramagnetic susceptibility. This decrease can be associated with the reduction of the effective $S_{z}$ by quantum fluctuations as well as with the decrease in the paramagnetic Weiss temperature (see below). Both effects become more noticeable for $H_{\perp} \geq 1 \mathrm{~T}$, as seen in Fig. 1.10.

Experiments show that $\chi_{z z}^{\prime}$ becomes independent of frequency, thus it reaches full equilibrium, for $\mu_{0} H_{\perp} \geq 2 \mathrm{~T}$. Above this field, $T_{\mathrm{b}} \leq 0.1 \mathrm{~K}$. Under these conditions, it is possible to explore the existence of a magnetic phase transition in $\mathrm{Fe}_{8}$ and study its critical behavior. As shown in Fig. $1.11(\mathrm{~A}), 1 / \chi_{z z}^{\prime}$ measured at $\mu_{0} H_{\perp}=2.25 \mathrm{~T}$ follows the Curie-Weiss law at sufficiently high $T$, becoming independent of $T$ below $0.34 \mathrm{~K}$, which we take as the critical temperature $T_{\mathrm{c}}$ at this field. Furthermore, the saturation value $1 / \chi_{\max }=9.5(5) \mathrm{cm}^{3} \mathrm{Oe} / \mathrm{emu}$ agrees well with the demagnetizing factor of our sample $\widetilde{N}_{z z}=10(1) \mathrm{cm}^{3} \mathrm{Oe} / \mathrm{emu}$. As discussed in section 1.2.2, this is the behavior expected for an equilibrium ferromagnetic phase transition. Additional evidence supporting the existence of a transition to a ferromagnetic phase is found in the results of neutron diffraction experiments, similar to those described above for the case of $\mathrm{Mn}_{12}$ acetate [49]. 
The ferromagnetic character of the low temperature phase agrees with theoretical predictions for the magnetic order resulting from dipole-dipole interactions $[7,109]$. However, to find out if this transition is dominantly driven by such interactions, one needs to compare also the experimental and theoretical values of $T_{\mathrm{c}}$. Unfortunately, equilibrium properties cannot be measured in $\mathrm{Fe}_{8}$ below $1 \mathrm{~K}$ for $H_{\perp}=0$. Yet, it is still possible to estimate $T_{\mathrm{c}} \simeq \theta$, using $\theta$ determined from the Curie-Weiss fit of the reciprocal susceptibility measured above $T_{\mathrm{b}}$. This method is particularly appropriate here, as the limiting value $1 / \chi_{\max }$ is known from experiments performed for $\mu_{0} H_{\perp} \geq 2 \mathrm{~T}$ (see, for instance, Fig. 1.11). It gives $T_{\mathrm{c}}\left(H_{\perp}=0\right)=0.60(5) \mathrm{K}$, in very good agreement with the theoretical $T_{\mathrm{c}}=0.54 \mathrm{~K}$ derived from Monte Carlo calculations [7]. It can be then be safely concluded that $\mathrm{Fe}_{8}$ becomes a pure dipolar ferromagnet at very low temperatures.

The reciprocal susceptibility shows a very similar behavior when $H_{\perp}$ is varied at constant $T$ (Fig. 1.11(B)). Again, $1 / \chi_{z z}^{\prime}$ depends linearly on $H_{\perp}$ until it saturates (to the same value $\simeq \widetilde{N}_{z z}$ ) below $\mu_{0} H_{\mathrm{c}}=2.65(5) \mathrm{T}$, which we take as the critical magnetic field at $T=110 \mathrm{mK}$. These experiments evidence that, also in $\mathrm{Fe}_{8}$, a sufficiently strong transverse magnetic field can destroy ferromagnetic order. However, as we shall see below, the nature of this transition is qualitatively different from that observed in $\mathrm{Mn}_{12}$ acetate. Before discussing this question in more detail, it is worth examining the critical behavior of the susceptibility, i.e. its temperature and field dependencies close to the phase transition.

The intrinsic susceptibility $\chi_{\mathrm{i}, \mathrm{zz}}^{\prime}$, corrected from demagnetizing effects, is plotted vs the reduced temperature $\left(T / T_{\mathrm{c}}-1\right)\left(\right.$ at $\left.\mu_{0} H_{\perp}=2.33 \mathrm{~T}\right)$ and field $\left(H_{\perp} / H_{\mathrm{c}}-1\right)$ (at $\left.T=110 \mathrm{mK}\right)$ in Fig. 1.12. Under equilibrium conditions, $\chi_{\mathrm{i}, \mathrm{zz}}^{\prime}$ should follow, as it approximately does, the power laws

$$
\chi_{\mathrm{i}, \mathrm{zz}}^{\prime}=\left(\frac{T-T_{\mathrm{c}}}{T_{\mathrm{c}}}\right)^{-\gamma_{\mathrm{cl}}}, \chi_{\mathrm{i}, \mathrm{zz}}^{\prime}=\left(\frac{H_{\perp}-H_{\mathrm{c}}}{H_{\mathrm{c}}}\right)^{-\gamma_{\mathrm{qu}}}
$$

The slopes give critical exponents $\gamma_{\mathrm{cl}}=1.1(1)$ and $\gamma_{\mathrm{qu}}=1.0(1)$, in good agreement with $\gamma=1$ of the mean-field universality class. This result agrees with the prediction that the marginal dimensionality for mean-field behavior is $d^{*}=3$ in an Ising dipolar ferromagnet [14] and with the fact that the critical exponents for the field-induced transition at $T \rightarrow 0$ become equivalent to those of the classical transition in $(d+1)$ dimensions [110].

The $H_{\mathrm{c}}-T_{c}$ magnetic phase diagram of $\mathrm{Fe}_{8}$ is shown in Fig 1.13. Each data point in this diagram was obtained by linearly extrapolating $1 / \chi_{z z}^{\prime}$, measured either as a function of temperature at a fixed $H_{\perp}$ or as a function of magnetic field at constant $T$. A third method, which provides equivalent results, consists of using the scaling plots of Fig. 1.12 to determine $T_{\mathrm{c}}$ independently. As expected, $T_{\mathrm{c}}$ decreases when quantum fluctuations increase, i.e. with increasing $H_{\perp}$, thus ferromagnetism survives only for sufficiently low temperatures or magnetic fields. 


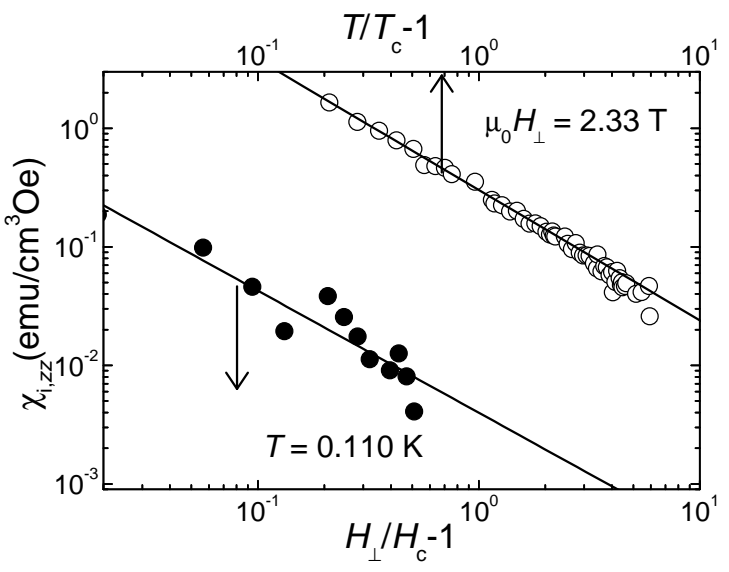

Fig. 1.12. Log-log plot of demagnetization-corrected $\chi_{\mathrm{i}, \mathrm{zz}}^{\prime}$ of $\mathrm{Fe}_{8}$ vs the reduced temperature (for $\mu_{0} H_{\perp}=2.33 \mathrm{~T}$ with $T_{c}=0.31 \mathrm{~K}, \circ$ ) and field (at $T=0.110 \mathrm{~K}$ with $\left.\mu_{0} H_{c}=2.65 \mathrm{~T}, \bullet\right)$. The linear fits give critical exponents $\gamma_{\mathrm{cl}} \simeq 1.1(1)$ and $\gamma_{\mathrm{qu}} \simeq 1.0(1)$

The experiments can be compared with predictions following from the $S=$ 10 quantum Ising model (1.7), using the fact that all anisotropy parameters as well as the magnetic field orientation are accurately known. As Fig. 1.13 shows, a very good fit is obtained for $J_{\mathrm{eff}} / k_{\mathrm{B}}=6 \times 10^{-3} \mathrm{~K}$, which, following Eq. (1.12), gives $T_{\mathrm{c}}$ equal to the experimental value of $0.6 \mathrm{~K}$ at zero field. Classical Monte Carlo simulations of the same model give, by contrast, the classical phase boundary shown by the dashed line in Fig. 1.13. This boundary is well approximated by $H_{\mathrm{c}}\left(T_{\mathrm{c}}\right)=H_{\mathrm{c}}(0)\left[1-T_{\mathrm{c}} / T_{c}\left(H_{\perp}=0\right)\right]^{1 / 2}$. In this model $H_{\mathrm{c}}(0)$ equals the anisotropy field $H_{K}=2\left[D-E\left(\sin ^{2} \phi-\cos ^{2} \phi\right)\right] / g \mu_{\mathrm{B}} S \simeq 3.8 \mathrm{~T}$, which clearly overestimates the experimental critical field due to the absence of quantum fluctuations. The existence of a quantum critical point in $\mathrm{Fe}_{8}$ can therefore be safely concluded. In summary, these results show that $\mathrm{Fe}_{8}$ provides a close approximation of the archetypical quantum Ising model in a transverse magnetic field. Recently, the magnetic field dependence of the high- $T$ susceptibility of $\mathrm{Mn}_{12}$-acetate- $\mathrm{MeOH}$ has also been found to be in agreement with the quantum Ising model [111]. This high symmetry $\mathrm{Mn}_{12^{-}}$ acetate variant has the same spin structure, anisotropy and similar lattice constants to the original $\mathrm{Mn}_{12}$-acetate but has minimal solvent disorder [111, 112]. An important implication of this study is that magnetic order in two chemically very similar SMMs can be described by distinct physical models. 




Fig. 1.13. $H_{\mathrm{c}}-T_{\mathrm{c}}$ phase diagram determined from the linear extrapolation of $1 / \chi^{\prime}$ to $1 / \chi_{\max } \cdot \bullet$ and $\circ$ correspond to $T_{\mathrm{c}}>T_{\mathrm{b}}$ and $T_{\mathrm{c}}<T_{\mathrm{b}}$, respectively; $\star$, data determined from susceptibility scaling plots, as those shown in Fig. 1.12. Solid line, quantum mean-field calculation of the phase boundary using Eq. (1.7) and the parameters given in the text; dashed line, classical phase diagram, derived from Monte Carlo simulations

\subsection{Conclusions and outlook}

The results reviewed in this chapter show that, despite their name, the physics of SMMs deviates from the image of an isolated molecule, especially at very low temperatures. In a crystal of SMMs, dipolar interactions induce the onset of long-range order. These materials provide therefore examples of pure dipolar magnets, of which so few exist in Nature.

Especially attractive are studies of long-range order in the presence of a transverse magnetic field, as those described in sections 1.5 and 1.6. The magnetic ground state results then from a subtle competition between dipolar couplings, quantum fluctuations, and random bias magnetic fields caused by molecular tilts, that is, by local disorder. The underlying physics is very rich, and depends qualitatively on the relative energy scales of these three interactions. In $\mathrm{Mn}_{12}$ acetate, random fields generated by molecular tilts dominantly suppresses ferromagnetism. By contrast, disorder-free $\mathrm{Fe}_{8}$ undergoes a quantum phase transition at $T \rightarrow 0$, purely induced by quantum fluctuations generated by the transverse magnetic field. Within this interpretation, $M_{z}$ vanishes above the critical field because the magnetic ground state becomes a quantum superposition of 'spin-up' and 'spin-down' states, a mesoscopic magnetic "Schrödinger's cat".

Molecular materials offer the possibility to realize in the lab two archetypical models, with broad interest for Magnetism and Solid State Physics: the random-field Ising model and the quantum Ising model. In this respect, molecular systems are appealing because properties such as the spin, magnetic 
anisotropy, and lattice symmetry can be controlled, to some extent, by chemical design. These possibility might enable experimentalists to explore situations which have not been realized yet, such as low-dimensional (i.e planes or chains) dipolar lattices, for which important deviations from the mean-field behavior can be expected [113], or situations with finite anisotropies that cannot be described by an Ising interaction Hamiltonian.

Quantum entanglement is enhanced near a quantum phase transition [114]. In molecular nanomagnets, the long-range character of the dominant dipolar interactions might lead to new sources of multipartite entanglement, thus change its range with respect to that found in spin systems with dominant nearest neighbor interactions. Entanglement is one of the resources for quantum computation and communication [115]. In my opinion, the measure and characterization of spin entanglement, and the study how quantum information propagates across a crystal of SMMs near the $T_{\mathrm{c}}-H_{\mathrm{c}}$ phase boundary provide fascinating, yet unexplored, topics for research.

The above considerations refer to equilibrium magnetic properties of molecular crystals. However, SMMs are famous for displaying fascinating dynamical phenomena, such as hysteresis, i.e. magnetic memory, and quantum spin tunneling. The experiments described in the present chapter, an a few others, suggest that pure quantum tunneling processes, despite their inherently temperature-independent character, are nevertheless able to bring the spin system into its thermal equilibrium state, be it paramagnetic or magnetically ordered. How this mechanism actually works and, in particular, how energy is exchanged between spins and phonons, is not clear yet and deserves to be investigated further. An interesting, related question is how magnetic correlations grow below $T_{\mathrm{c}}$, especially when spins are only able to flip by tunneling. This question has been addressed by Monte Carlo simulations [32] performed on the basis of the Prokof'ev and Stamp model for pure quantum tunneling [31], but needs to be tested experimentally. Another relatively unexplored area, especially from the experimental point of view, refers to the structure of domain walls in dipolar ferromagnets and their classical or quantum dynamics.

Close to a phase transition, the system dynamics tends to suffer from a "critical slowing down" [116]. The study of such non-equilibrium critical phenomena came to the fore when it was shown that they give information on the formation and the structure of defects in the early Universe and that some experiments could be carried out on real systems available at the laboratory [116]. An example is the formation of vortices in the vicinity of the (classical) phase transition of superfluid Helium at $T_{\lambda}=2.14 \mathrm{~K}$ [117]. Crystals of SMMs offer the possibility to investigate non-equilibrium spin dynamics (e.g. the nucleation of domain walls) across a quantum critical point $\left(T \simeq 0, H_{\mathrm{c}}\right)$. These studies can reveal the influence of quantum fluctuations [118] and might be relevant to the implementation of adiabatic quantum computation schemes [119] in crystals of SMMs. 


\section{8 acknowledgments}

I would like to express my deep gratitude to the co-editors of this book J. Bartolomé and J. F. Fernández, who also happened to be my thesis co-advisers, for introducing me to the physics of molecular nanomagnets, almost twenty years ago, and for their continued support and inspiration. Several highly esteemed colleagues have contributed to the work reported in this chapter. Many thanks to (in alphabetical order) G. Aromí, O. N. Bakharev, R. Ballou, B. Barbara, E. Bouwman, H. B. Brom, E. Burzurí, J. Campo, Ch. Carbonera, G. Christou, M. Evangelisti, J. F. Fernández, J. Gómez, L. J. de Jongh, J. Krzystek, J. Luzón, G. J. McIntyre, F. L. Mettes, S. Maegawa, O. Montero, A. Morello, E. Ressouche, D. Ruiz-Molina, C. Schlegel, J. van Slageren, S. Tanase, S. J. Teat, Tomoaki Yamasaki and Miki Ueda. I am also grateful to A. Kent and P. Subedi for their valuable comments and for sharing with me their theoretical predictions for the magnetic phase diagram of $\mathrm{Mn}_{12}$ acetate in the presence of interstitial disorder [50], which are shown in Fig. 1.9.

\section{References}

1. J. H. van Vleck, Phys. Rev. 74, 1168 (1948)

2. C. Kittel, Rev. Mod. Phys. 21, 541 (1949)

3. J. A. Sauer, Phys. Rev. 57, 142 (1940)

4. J. M. Luttinger and L. Tisza, Phys. Rev. 70, 954 (1946)

5. Th. Niemeijer and H. W. J. Blöte, Physica 67, 125 (1973)

6. S. K. Misra, Phys. Rev. B 14, 5065 (1976)

7. J. F. Fernández and J. J. Alonso, Phys. Rev. B 62, 53 (2000); ibid 65, 189901(E) (2000)

8. H.-J. Xu, B. Bergersen, and Z. Rácz, J. Phys. Condens. Matter 4, 2035 (1992)

9. J. P. Bouchaud and P. G. Zérah, Phys. Rev. B 47, 9095 (1993)

10. A. J. Millis, A. D. Kent, M. P. Sarachik, and Y. Yeshurun, Phys. Rev. B 81, $024423(2010)$

11. R. B. Griffiths, Phys. Rev. 176, 655 (1968)

12. C. Kittel, Phys. Rev. 82, 965 (1951).

13. M. H. Cohen and F. Keffer, Phys. Rev. 99, 1135 (1955)

14. A. Aharony, Phys. Rev. B 8, 3363 (1973).

15. A. H. Cooke, D. A. Jones, J. F. A. Silva, and M. R. Wells, J. Phys. C: Solid State Phys. 8, 4083 (1975)

16. L. M. Holmes, J. Als-Nielsen, and H. J. Guggenheim, Phys. Rev. B 12, 180 (1975)

17. J. Als-Nielsen, Phys. Rev. Lett. 37, 1161 (1976)

18. G. Mennenga, L. J. de Jongh, and W. J. Huiskamp, J. Mag. Mag. Mater. 44, $59(1984)$

19. D. H. Reich, T. F. Rosenbaum, G. Aeppli, and H. J. Guggenheim, Phys. Rev. B 34, 4956 (1986)

20. M. R. Roser and L. R. Corruccini, Phys. Rev. Lett. 65, 1064 (1990)

21. M. R. Roser, J. Xu, S. J. White, and L. R. Corruccini, Phys. Rev. B 45, 12337 (1992) 
22. S. J. White, M. R. Roser, J. Xu, J. T. van der Noorda, and L. R. Corruccini, Phys. Rev. Lett. 71, 3553 (1993)

23. H. M. Ronnow et al., Science 308, 389 (2005)

24. M. Schechter and P. C. E. Stamp, Phys. Rev. B 78, 054438 (2008)

25. R. Sessoli, D. Gatteschi, A. Caneschi, and M. A. Novak, Nature (London) 365, 141 (1993)

26. D. Gatteschi, A. Caneschi, L. Pardi, and R. Sessoli, Science 265, 1054 (1994)

27. G. Christou, D. Gatteschi, D. N. Hendrickson, and R. Sessoli, MRS Bull. 25, $26(2000)$

28. D. Gatteschi and R. Sessoli, Angew. Chem. Int. Ed. 42, 268 (2003)

29. D. Gatteschi, R. Sessoli and J. Villain, Molecular Nanomagnets, 1st edn. (Oxford University Press, Oxford, 2006)

30. R. B. Stinchcombe, J. Phys. C: Solid State Phys. 6, 2459 (1973)

31. N. V. Prokof'ev and P. C. E. Stamp, Phys. Rev. Lett. 80, 5794 (1998)

32. J. F. Fernández, Phys. Rev. B 66, 064423 (2002)

33. J. R. Friedman, M. P. Sarachik, J. Tejada, and R. Ziolo, Phys. Rev. Lett. 76, 3830 (1996)

34. J. M. Hernández, X. X. Zhang, F. Luis, J. Bartolomé, J. Tejada, and R. Ziolo, Europhys. Lett. 35, 301 (1996)

35. L. Thomas, F. Lionti, R. Ballou, D. Gatteschi, R. Sessoli, B. Barbara, Nature 383, 145 (1996)

36. C. Sangregorio, T. Ohm, C. Paulsen, R. Sessoli, and D. Gatteschi, Phys. Rev. Lett. 78, 4645 (1997)

37. W. Wernsdorfer and R. Sessoli, Science 284, 133 (1999)

38. E. del Barco, N. Vernier, J. M. Hernández, J. Tejada, E. M. Chudnovsky, E. Molins, and G. Bellessa, Eurphys. Lett. 47, 722 (1999)

39. F. Luis, F. L. Mettes, J. Tejada, D. Gatteschi, and L. J. de Jongh, Phys. Rev. Lett. 85, 4377 (2000)

40. J. A. Hertz, Phys. Rev. B 14, 1165 (1976).

41. S. Sachdev, Quantum Phase Transitions, Cambridge Univ. Press (Cambridge, 1999).

42. S. Morup, M. F. Hansen, and C. Frandsen, Beilstein J. Nanotechnology 1, 182 (2010)

43. M. Evangelisti and E. K. Brechin, Dalton Trans. 39, 4672 (2010)

44. A. Morello, F. L. Mettes, F. Luis, J. F. Fernández, J. Krzystek, G. Aromí, G. Christou, and L. J. de Jongh, Phys. Rev. Lett. 90, 017206 (2003).

45. M. Evangelisti, F. Luis, F. L. Mettes, N. Aliaga, G. Aromí, J. J. Alonso, G. Christou, and L. J. de Jongh, LJ, Phys. Rev. Lett. 93, 117202 (2004)

46. F. Luis, J. Campo, J. Gómez, G. J. McIntyre, J. Luzón, and D. Ruiz-Molina, Phys. Rev. Lett. 95, 227202 (2005)

47. A. Morello, F. L. Mettes, O. N. Bakharev, H. B. Brom, L. J. de Jongh, F. Luis, J. F. Fernández, and G. Aromí, Phys. Rev. B 73, 134406 (2006)

48. G. Aromí, E. Bouwman, E. Burzurí, Ch. Carbonera, J. Krzystek, F. Luis, C. Schlegel, J. van Slageren, S. Tanase, and S. J. Teat, Chemistry A Eur. J. 14, 11158 (2008)

49. E. Burzurí, F. Luis, B. Barbara, R. Ballou, E. Ressouche, O. Montero, J. Campo, and S. Maegawa, Phys. Rev. Lett. 107, 097203 (2011)

50. B. Wen, P. Subedi, L. Bo, Y. Yeshurun, M. P. Sarachik, A. D. Kent, A. J. Millis, C. Lampropoulos, and G. Christou, Phys. Rev. B 82, 014406 (2010) 
51. L. J. de Jongh and A. R. Miedema, Adv. Phys. 23, 1 (1974)

52. A. Aharoni Introduction to the Theory of Ferromagnetism, 2nd edn. (Oxford University Press, Oxford, 2000)

53. D. A. Garanin, Phys. Rev. B 81, 220408(R) (2010)

54. S. Li, L. Bo, B. Wen, M. P. Sarachik, P. Subedi, A. D. Kent, Y. Yeshurun, A. J. Millis, C. Lampropoulos, S. Mukherjee, and G. Christou, Phys. Rev. B 82, $174405(2010)$

55. R. de L. Kronig, Physica 6, 33 (1939)

56. J. H. Van Vleck, Phys. Rev. 57, 426 (1940)

57. R. Orbach, Proc. Roy. Soc. (London) A264, 456 (1961); R. Orbach, Proc. Roy. Soc. (London) A264, 485 (1961)

58. J. Villain, F. Hartman-Boutron, R. Sessoli, and A. Rettori, Europhys. Lett. 27, $159(1994)$

59. D. A. Garanin and E. M. Chudnovsky, Phys. Rev. B 56, 11102 (1997)

60. F. Luis, J. Bartolomé, and J. F. Fernández, Phys. Rev. B 57,505 (1998)

61. A. Fort, A. Rettori, J. Villain, D. Gatteschi, and R. Sessoli, Phys. Rev. Lett. 80 $612(1998)$

62. M. N. Leuenberger, and D. Loss, Phys. Rev. B 61, 1286 (2000)

63. L. Thomas, A. Caneschi, and B. Barbara, Phys. Rev. Lett. 83, 2398 (1999)

64. M. Evangelisti, F. Luis, F. L. Mettes, R. Sessoli, and L. J. de Jongh, Phys. Rev. Lett. 95, 227206 (2005)

65. M. Evangelisti, A. Candini, A. Ghirri, M. Affronte, G. W. Powell, I. A. Gass, P. A. Wood, S. Parsons, E. K. Brechin, D. Collison, and S. L. Heath, Phys. Rev. Lett. 97, 167202 (2006)

66. C. Vecchini, D. H. Ryan, L. M. D. Cranswick, M. Evangelisti, W. Kockelmann, P. G. Radaelli, A. Candini, M. Affronte, I. A. Gass, E. K. Brechin, and O. Moze, Phys. Rev. B 77, 224403 (2008)

67. N. V. Prokof'ev and P. C. E. Stamp, Rep. Progr. Phys. 63, 669 (2000)

68. J. F. Fernández and J. J. Alonso, Phys. Rev. Lett. 91, 047202 (2003); see also I. S. Tupitsyn and P. C. E. Stamp, ibid 92, 119701 (2004) and J. F. Fernández and J. J. Alonso, ibid 92, 119702 (2004)

69. J. F. Fernández and J. J. Alonso, Phys. Rev. B 69, 024411 (2004)

70. D. A. Garanin, J. Phys. A: Math. Gen. 24, L61 (1991).

71. W. Wernsdorfer, M. Murugesu, and G. Christou, Phys. Rev. Lett. 96, 057208 (2006)

72. S. McHugh, R. Jaafar, M. P. Sarachik, Y. Myasoedov, H. Shtrikman, E. Zeldov, R. Bagai, and G. Christou, Phys. Rev. B 79, 052404 (2009)

73. D. Ruiz-Molina, Z. S. Sun, B. Albela, K. Folting, J. Ribas, G. Christou, and D. N. Hendrickson, Angew. Chem., Int. Ed. 37, 300 (1998)

74. G. Aromí, M. J. Knapp. J.-P. Claude, J. C. Huffman, D. N. Hendrickson, and G. Christou, J. Am. Chem. Soc. 121, 5489 (1999)

75. E. Burzurí, Dissertation, University of Zaragoza (2011)

76. D. A. Garanin and E. M. Chudnovsky, Phys. Rev. B 78, 174425 (2008)

77. A. Morello, O. N. Bakharev, H. B. Brom, R. Sessoli and L. J. de Jongh, Phys. Rev. Lett. 93, 197202 (2004).

78. F. Luis, M. J. Martínez-Pérez, O. Montero, E. Coronado, S. Cardona-Serra, C. Martí-Gastaldo, J. M. Clemente-Juan, J. Ses, D. Drung, and T. Schurig, Phys. Rev. B 82, 060403(R) (2010) 
79. S. M. J. Aubin, N. R. Dilley, L. Pardi, J. Krzystek, M. W. Wemple, L. C. Brunel, M. B. Maple, G. Christou, and D. N. Hendrickson, J. Am. Chem. Soc. 120, 4991 (1998)

80. N. Aliaga, K. Folting, D. N. Hendrickson, and G. Christou, Polyhedron 20, 1273 (2001)

81. T. Kadowaki and H. Nishimori, Phys. Rev. E 58, 5355 (1998).

82. J. Brooke et al., Science 284, 779 (1999).

83. F. L. Mettes, F. Luis and L. J. de Jongh, Phys. Rev. B 64, 174411 (2001)

84. S. Sachdev and J. Ye, Phys. Rev. Lett. 69, 2411 (1992).

85. A. V. Chubukov and S. Sachdev, Phys. Rev. Lett. 71, 169 (1993).

86. A. Sokol and D. Pines, Phys. Rev. Lett. 71, 2813 (1993).

87. Q. Si and F. Steglich, Science 329, 1161 (2010).

88. S. A. Carter, T. F. Rosenbaum, J. M. Honig, and J. Spalek, Phys. Rev. Lett. 67, 3440 (1991)

89. D. Bitko, T. F. Rosenbaum and G. Aeppli , Phys. Rev. Lett. 77, 940 (1996)

90. A. Yeh et al., Nature 419, 459-462 (2002)

91. P. Stasiak and M. J. P. Gingras, Phys. Rev. B 78, 224412 (2008)

92. T. Lis, Acta Crystallogr. B 36, 2042 (1980)

93. R. A. Robinson, P. J. Brown, D. N. Argyriou, D. N. Hendrickson, and S. M. J. Aubin, J. Phys. Condens. Mater 12, 2805 (2000)

94. A. L. Barra, D. Gatteschi, and R. Sessoli, Phys. Rev. B 56, 8192 (1997)

95. S. Hill et al., Phys. Rev. Lett. 80, 2453 (1998)

96. I. Mirebeau et al., Phys. Rev. Lett. 83, 628 (1999)

97. S. Hill, R. S. Edwards, S. I. Jones, N. S. Dalal, J. M. North, Phys. Rev. Lett. 90, $217204(2003)$

98. S. Takahashi, R. S. Edwards, J. M. North, S. Hill, and N. S. Dalal, Phys. Rev. B 70, 094429 (2004)

99. R. Bircher, G. Chaboussant, A. Sieber, H. U. Gudel, and H. Mutka, Phys. Rev. B 70, 212413 (2004)

100. A. Cornia, R. Sessoli, L. Sorace, D. Gatteschi, A. L. Barra, and C. Daiguebonne, Phys. Rev. Lett. 89, 257201 (2002)

101. E. del Barco, A. D. Kent, E. M. Rumberger, D. N. Hendrickson, and G. Christou, Phys. Rev. Lett. 91, 047203 (2003)

102. E. del Barco, A. D. Kent, S. Hill, J. M. North, N.S. Dalal, E. M. Rumberger, D. N. Hendrickson, N. Chakov, and G. Christou, Journal of Low Temperature Physics 140, 119 (2005)

103. E. Burzurí, Ch. Carbonera, F. Luis, D. Ruiz-Molina, C. Lampropoulos, and G. Christou, Phys. Rev. B 80, 224428 (2009)

104. K. Park, T. Baruah, N. Bernstein, and M. R. Pederson, Phys. Rev. B 69, $144426(2004)$

105. K. Wieghardt, K. Pohl, I. Jibril, and G. Huttner, Angew. Chem. Int. Ed. 23 $77(1984)$

106. A. L Barra et al., Europhys. Lett. 35, 133 (1996)

107. R. Caciuffo et al., Phys. Rev. Lett. 81, 4744 (1998)

108. M. Ueda et al., J. Phys. Soc. Jpn. 70, 3084 (2001)

109. X. Martínez-Hidalgo, E. M. Chudnovsky, and A. Aharony, Europhys. Lett. 55, $273(2001)$

110. R. J. Elliot, P. Pfeuty, and C. Wood, Phys. Rev. Lett. 25, 443 (1970).

111. P. Subedi, A. D. Kent, B. Wen, M. P. Sarachik, Y. Yeshurun, A. J. Millis, S. Mukherjee, and G. Christou, Phys. Rev. B 85, 134441 (2012) 
112. G. Redler, C. Lampropoulos, S. Datta, C. Koo, T. C. Stamatatos, N. E. Chakov, G. Christou, and S. Hill, Phys. Rev. B 80, 094408 (2009)

113. J. F. Fernández and J. J. Alonso, Phys. Rev. B 76, 014403 (2007)

114. A. Osterloh, L. Amico, G. Falci, and R. Fazio, Nature 416, 608 (2002)

115. M. A. Nielsen and I. L. Chuang, Quantum Computation and Quantum Information, 1st edn. (Cambridge University Press, Cambridge, 2000)

116. W. H. Zurek, Nature 317, 505 (1985)

117. P. C. Hendry, N. S. Lawson, R. Lee, P. Mc Clintok, and C. Williams, Nature 368, 315 (1994)

118. W. H. Zurek, U. Dorner, and P. Zoller, Phys. Rev. Lett. 95, 105701 (2005)

119. E. Farhi, J. Goldstone, S. Gutmann, J. Lapan, A. Lundgren, and D. Preda, Science 292, 472 (2001) 